\title{
The Oxidative Mannich Reaction Catalyzed by Dirhodium Caprolactamate
}

Arthur J. Catino, Jason M. Nichols, Brian J. Nettles, and Michael P. Doyle

Department of Chemistry and Biochemistry

The University of Maryland

College Park, MD 20742

\section{Supporting Information}

(17 pages) 
General. All products were characterized and in agreement with those previously described. Yields reported are for isolated yields unless otherwise noted. tert-Butyl hydroperoxide (T-HYDRO ${ }^{\circledR}, 70 \mathrm{wt}$ \% in $\mathrm{H}_{2} \mathrm{O}$ ) was obtained from Aldrich and used as received. The preparation of dirhodium(II) caprolactamate $\left[\mathrm{Rh}_{2}(\mathrm{cap})_{4} \cdot 2 \mathrm{CH}_{3} \mathrm{CN}\right]$ has been previously described. ${ }^{1}$ 2-Triisopropylsiloxyfurans were prepared according to the procedure of Martin. ${ }^{2}$ 5-Allyl-2-triisopropylsiloxyfuran was prepared according to the procedure of Liras. ${ }^{3}$ Amines (Table 2, entries 2, 3, and 7) were prepared via reductive amination with $\mathrm{NaCNBH}_{3}-\mathrm{ZnCl}_{2}$ according to the procedure of Kim, et $a l^{4}{ }^{4}$ 2- $N, N$-Dimethylaminostyrene was prepared via Wittig reaction from the known 2-N,N-dimethylaminobenzaldehyde.${ }^{5} N$-Ethyl- $N$-methylaniline (Table 2, entry 6) was obtained from the $\mathrm{NaBH}_{4}-\mathrm{I}_{2}$ reduction of $N$-methylacetanilide. ${ }^{6} \quad N$-Phenyl-1,2,3,4-tetrahydroisoquinoline was prepared according to the procedure of Quach. ${ }^{7}$ Preparative chromatographic purification was performed using SiliCycle (60尺, 40-63 mesh) silica gel according to the method of Still. ${ }^{8}$ Thin layer chromatography (TLC) was performed on Merck $0.25 \mathrm{~mm}$ silica gel $60 \mathrm{~F}_{254}$ plates with visualization by fluorescence quenching. ${ }^{1} \mathrm{H}$ NMR (400 MHz) and ${ }^{13} \mathrm{C}$ NMR (100 MHz) spectra were obtained on a Bruker DRX-400 NMR as solutions in $\mathrm{CDCl}_{3}$. Chemical shifts are reported in parts per million (ppm), $\delta$ downfield from $\mathrm{Me}_{4} \mathrm{Si}$ (TMS); coupling constants are reported in Hertz (Hz).

Representative Procedure. T-HYDRO ${ }^{\circledR}$ (1.2 equiv) was added in one portion to a stirring solution of amine (2.0 equiv), 2-triisopropoxysilylfuran (1.0 equiv), and $\mathrm{Rh}_{2}$ (cap) $)_{4}(1.0 \mathrm{~mol} \%)$ in $\mathrm{MeOH}(0.27 \mathrm{M} /$ [siloxyfuran]). The reaction mixture was heated at $60^{\circ} \mathrm{C}$ for $3-5 \mathrm{hr}$ (or $16 \mathrm{hr}$ with $0.1 \mathrm{~mol} \%$ catalyst). The solvent was then evaporated and the product was purified directly on silica gel.

\footnotetext{
${ }^{1}$ Doyle, M. P.; Westrum, L. J.; Wolthuis, W. N. E.; See, M. M.; Boone, W. P.; Bagheri, V.; Pearson, M. M. J. Am. Chem. Soc. 1993, 115, 958.

${ }^{2}$ Bur, S. K.; Martin, S. F. Tetrahedron 2001, 57, 3221 and references therein.

${ }^{3}$ Liras, S.; Davoren, J. E.; Bordner, J. Org. Lett. 2001, 3, 703.

${ }^{4}$ Kim, S.; Oh, C. H.; Ko, J. S.; Ahn, K. H.; Kim, Y. J. J. Org. Chem. 1985, 50, 1927.

${ }^{5}$ Stanetty, P.; Rodler, I. K.; Krumpak, B. J. Prakt. Chem. 1994, 336, 333.

${ }^{6}$ Prasad, A. S. B.; Kanth, J. V. B.; Periasamy, M. Tetrahedron 1992, 48, 4623.

${ }^{7}$ Quach, T. D.; Batey, R. A. Org. Lett. 2003, 5, 4397.

${ }^{8}$ Still, W. C.; Kahn, M.; Mitra, A. J. J. Org. Chem. 1978, 43, 2923.
} 


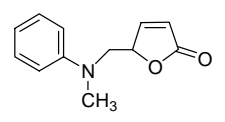

Scheme 1, 4: Purified by chromatography on silica gel $(5: 1 \rightarrow 1: 1$ hexanes/EtOAc); orange oil: $\mathrm{TLC} \mathrm{R}_{f}=0.30\left(1: 1\right.$ hexanes/EtOAc); ${ }^{1} \mathrm{H} \mathrm{NMR}\left(400 \mathrm{MHz}, \mathrm{CDCl}_{3}\right) \delta 7.47$ (dd, $J=5.8,1.5 \mathrm{~Hz}, 1 \mathrm{H}), 7.25$ (t, $J=8.0 \mathrm{~Hz}, 2 \mathrm{H}), 6.77(\mathrm{t}, J=7.3 \mathrm{~Hz}, 1 \mathrm{H}), 6.72(\mathrm{~d}, J=8.0 \mathrm{~Hz}, 2 \mathrm{H}), 6.13$ (dd, $J=5.6,2.1 \mathrm{~Hz}, 1 \mathrm{H}), 5.27(\mathrm{tt}, J=5.8,1.8 \mathrm{~Hz}, 1 \mathrm{H}), 3.69(\mathrm{~d}, J=6.0 \mathrm{~Hz}, 2 \mathrm{H}), 3.02(\mathrm{~s}, 3 \mathrm{H}) ;{ }^{13} \mathrm{C} \mathrm{NMR}$ $(100 \mathrm{MHz}) \delta 172.6,154.4,148.2,129.4,122.2,117.4,112.3,81.9,55.0,39.5$; IR (neat) $1755(\mathrm{C}=\mathrm{O}) \mathrm{cm}^{-1}$; HRMS (EI) calcd for $\mathrm{C}_{12} \mathrm{H}_{14} \mathrm{NO}_{2} 204.1025$, found $204.1026(\mathrm{M}+\mathrm{H})$.
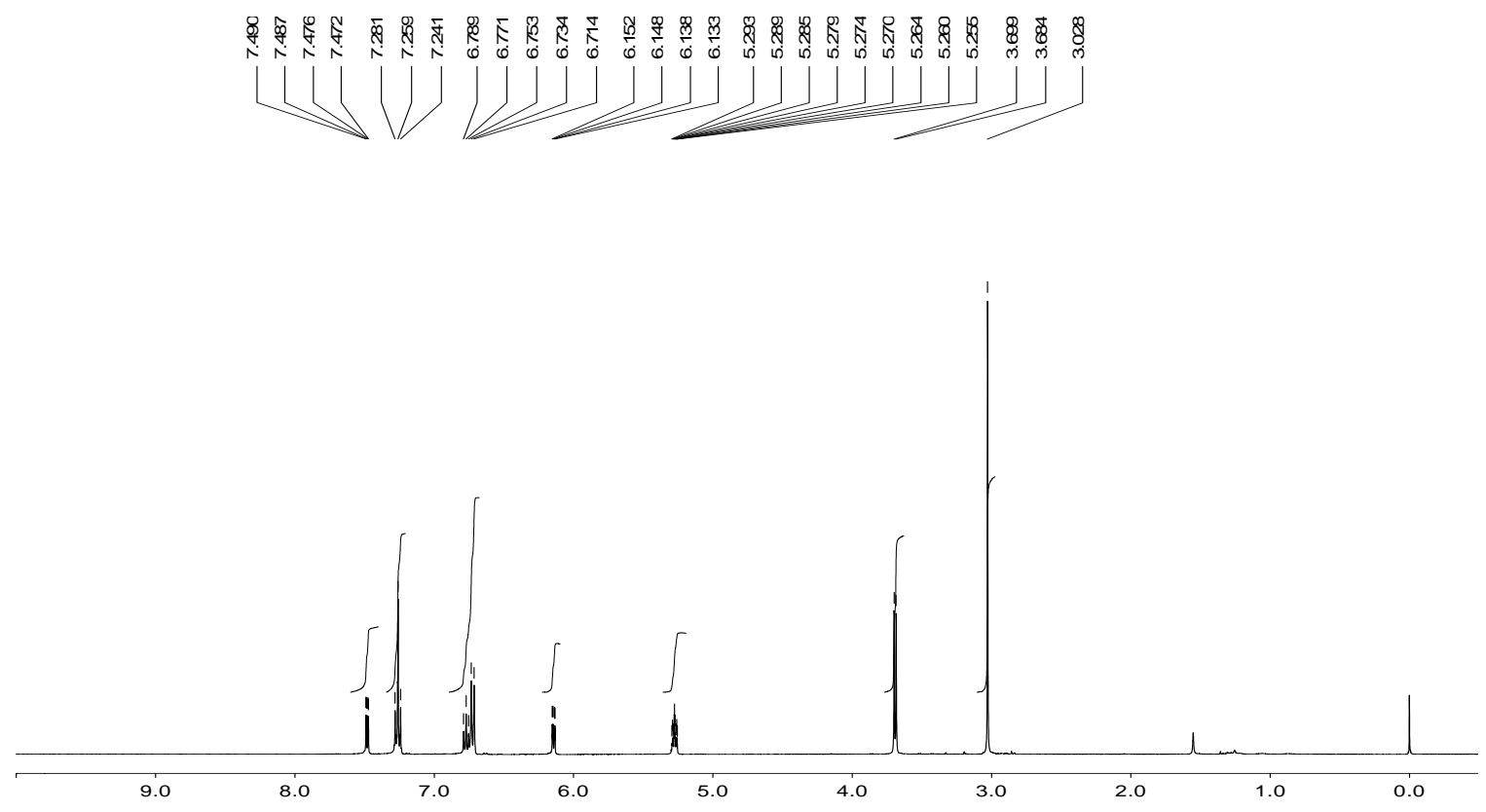
ppm (t1)

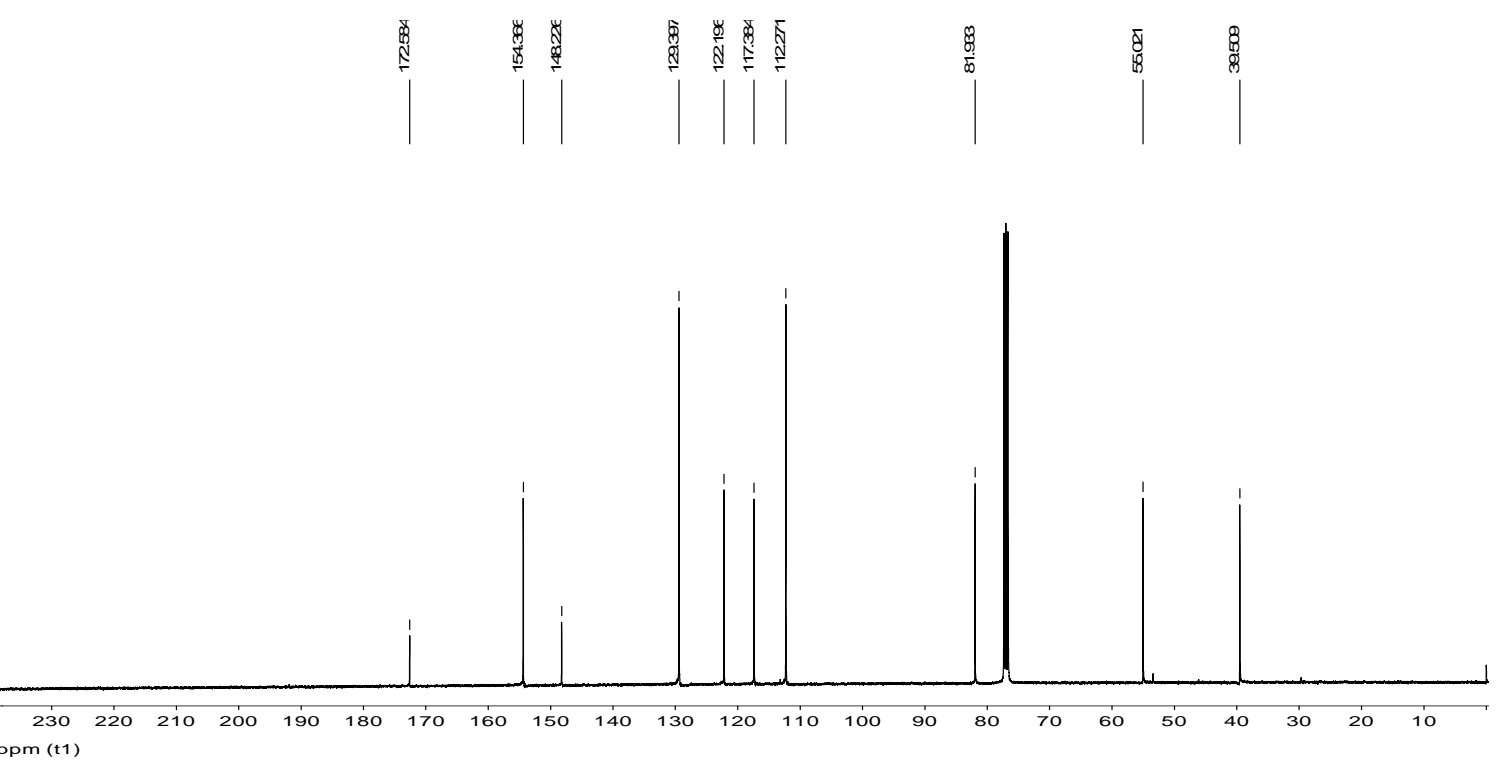




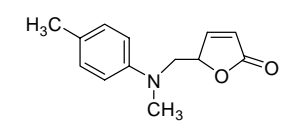

Table 2, entry $1\left(\mathrm{R}=\mathrm{CH}_{3}\right)$ : Purified by chromatography on silica gel $\left(5: 1 \rightarrow 1: 1\right.$ hexanes/EtOAc); orange oil: $\mathrm{TLC} \mathrm{R}_{f}=0.50\left(1: 1\right.$ hexanes/EtOAc); ${ }^{1} \mathrm{H} \mathrm{NMR}\left(400 \mathrm{MHz}, \mathrm{CDCl}_{3}\right)$ $\delta 7.47(\mathrm{~d}, J=5.6 \mathrm{~Hz}, 1 \mathrm{H}), 7.06(\mathrm{~d}, J=7.4 \mathrm{~Hz}, 2 \mathrm{H}), 6.64(\mathrm{~d}, J=8.1,2 \mathrm{H}), 6.11(\mathrm{~d}, J=5.4 \mathrm{~Hz}, 1 \mathrm{H}), 5.26-$ $5.24(\mathrm{~m}, 1 \mathrm{H}), 3.64$ (d, $J=5.8 \mathrm{~Hz}, 2 \mathrm{H}), 2.99$ (s, 3H), $2.26(\mathrm{~s}, 3 \mathrm{H}) ;{ }^{13} \mathrm{C} \mathrm{NMR}(100 \mathrm{MHz}) \delta 172.6,154.5$, 146.2, 129.9, 126.7, 122.0, 112.6, 82.0, 55.3, 39.6, 20.1; IR (neat) $1751(\mathrm{C}=\mathrm{O}) \mathrm{cm}^{-1}$; HRMS (EI) calcd for $\mathrm{C}_{13} \mathrm{H}_{15} \mathrm{NO}_{2} 218.1181$, found $218.1176(\mathrm{M}+\mathrm{H})$.
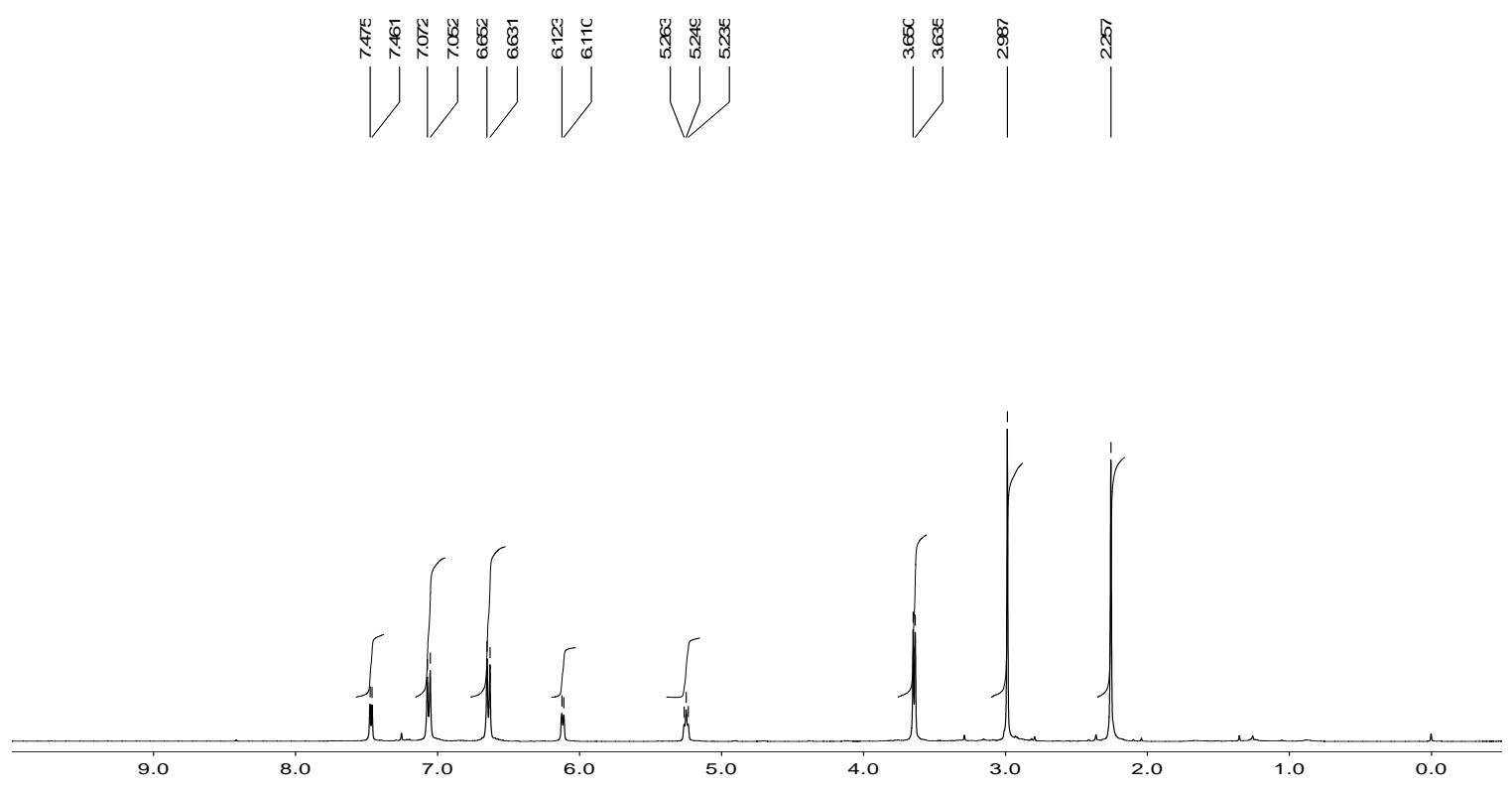

ppm (t1)
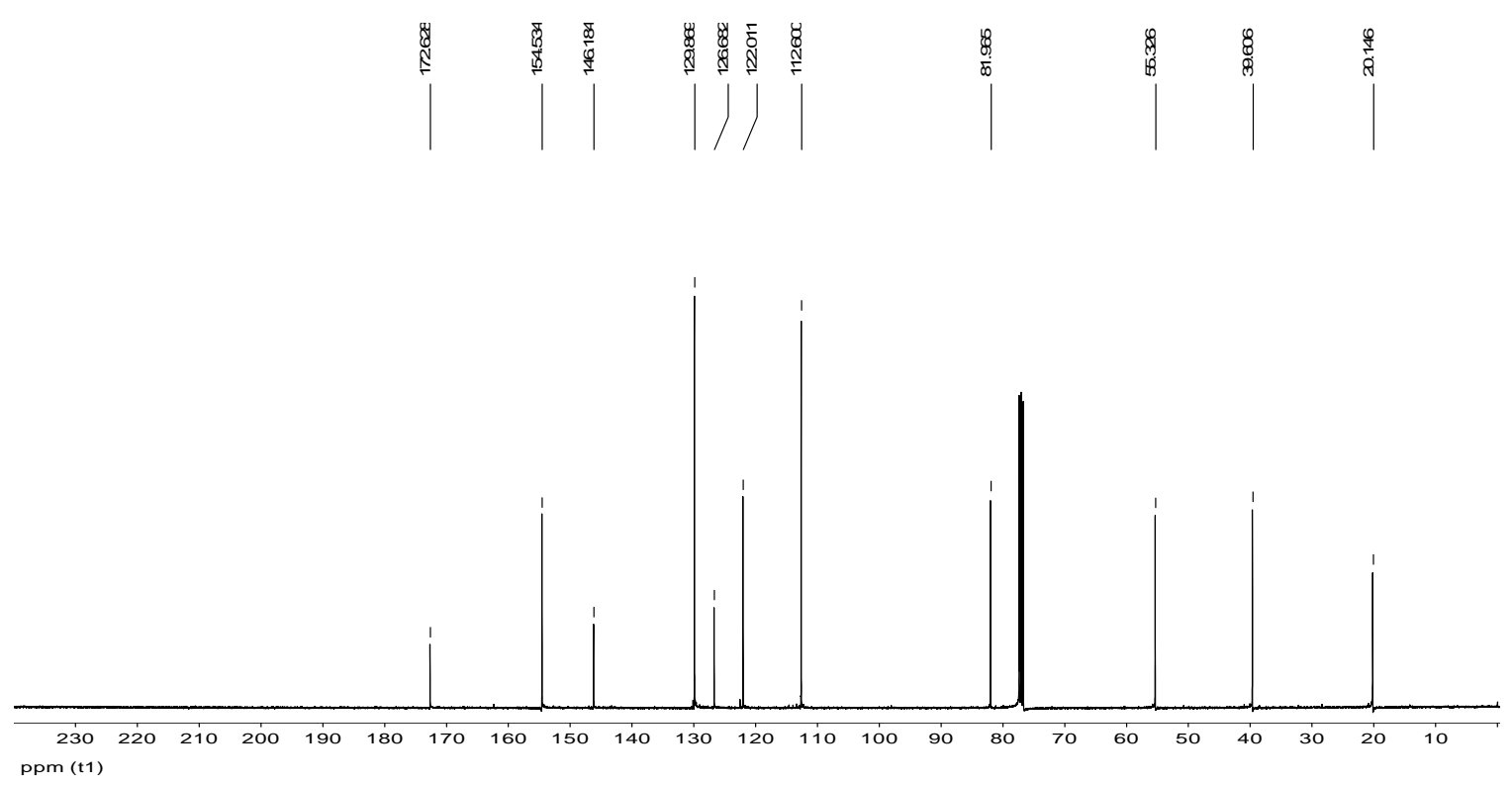


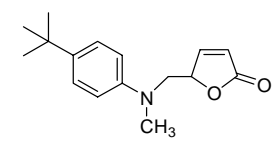

Table 2, entry $1(\mathrm{R}=t$-Bu): Purified by chromatography on silica gel $\left(5: 1 \rightarrow 1: 1\right.$ hexanes/EtOAc); orange oil: TLC $_{f}=0.25(2: 1$ hexanes/EtOAc $) ;{ }^{1} \mathrm{H}$ NMR $\left(400 \mathrm{MHz}, \mathrm{CDCl}_{3}\right)$ $\delta 7.48(\mathrm{~d}, J=5.6 \mathrm{~Hz}, 1 \mathrm{H}), 7.26(\mathrm{~d}, J=8.7 \mathrm{~Hz}, 2 \mathrm{H}), 6.66(\mathrm{~d}, J=8.7 \mathrm{~Hz}, 2 \mathrm{H}), 6.10-6.08(\mathrm{~m}, 1 \mathrm{H}), 5.25-$ $5.22(\mathrm{~m}, 1 \mathrm{H}), 3.67-3.56(\mathrm{~m}, 2 \mathrm{H}), 2.97(\mathrm{~s}, 3 \mathrm{H}), 1.28(\mathrm{~s}, 9 \mathrm{H}) ;{ }^{13} \mathrm{C} \mathrm{NMR}(100 \mathrm{MHz}) \delta 172.5,154.6,145.9$, 139.8, 125.9, 121.8, 111.9, 81.9, 55.1, 39.3, 33.5, 31.3; IR (neat) $1756(\mathrm{C}=\mathrm{O}) \mathrm{cm}^{-1}$; HRMS (EI) calcd for $\mathrm{C}_{16} \mathrm{H}_{22} \mathrm{NO}_{2} 260.1651$, found $260.1646(\mathrm{M}+\mathrm{H})$.

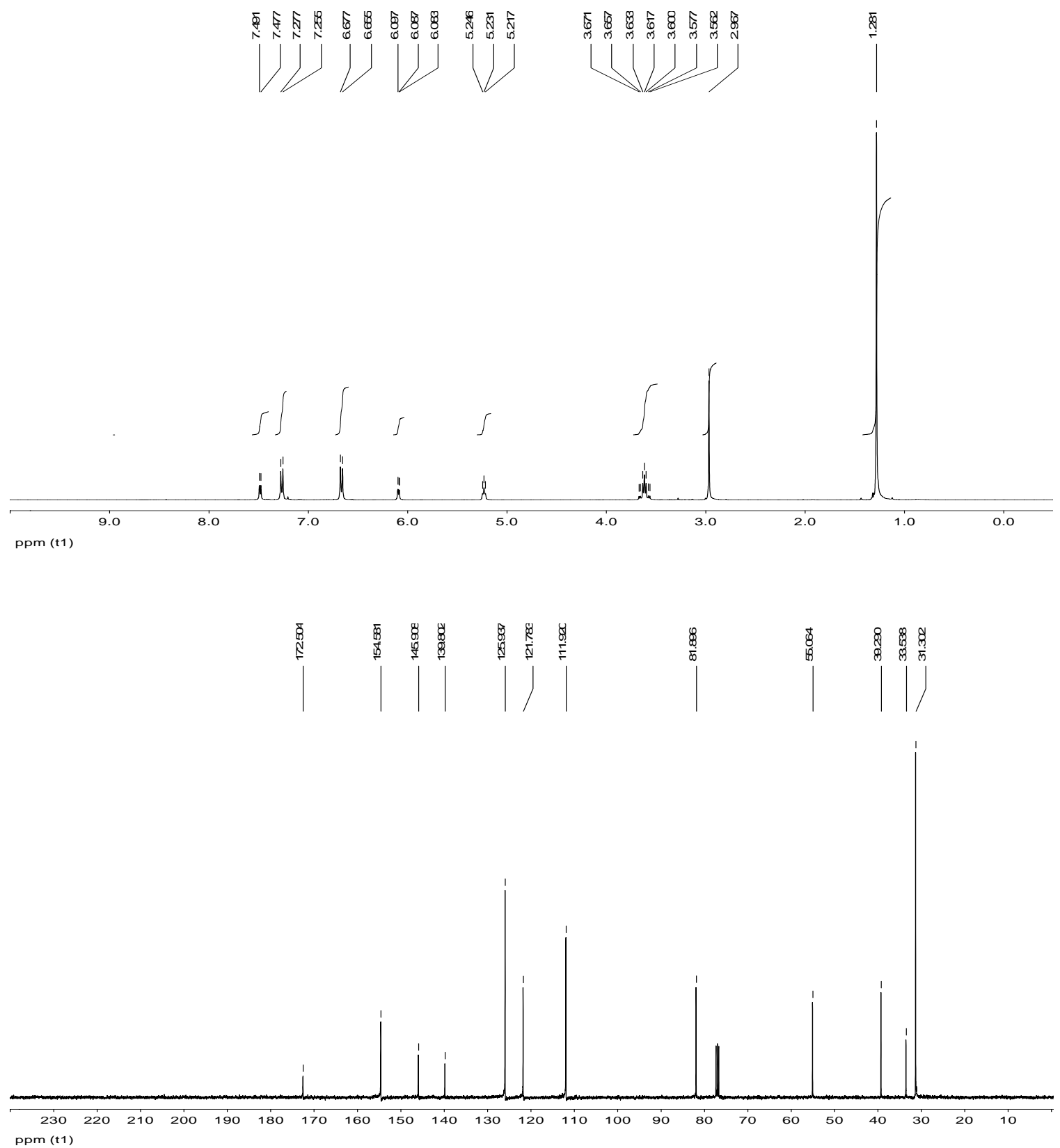




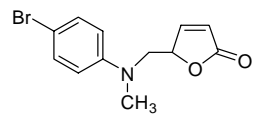

Table 2, entry $1(\mathrm{R}=\mathrm{Br})$ : Purified by chromatography on silica gel $\left(5: 1 \rightarrow\right.$ 1:1 hexanes/EtOAc); yellow oil: $\mathrm{TLC} \mathrm{R}_{f}=0.22\left(2: 1\right.$ hexanes/EtOAc); ${ }^{1} \mathrm{H}$ NMR $\left(400 \mathrm{MHz}, \mathrm{CDCl}_{3}\right)$ $\delta 7.45(\mathrm{dd}, J=5.8,1.3 \mathrm{~Hz}, 1 \mathrm{H}), 7.32(\mathrm{~d}, J=8.9 \mathrm{~Hz}, 2 \mathrm{H}), 6.58(\mathrm{~d}, J=8.9 \mathrm{~Hz}, 2 \mathrm{H}), 6.14$ (dd, $J=5.7$, $1.9 \mathrm{~Hz}$ ), $5.26-5.23(\mathrm{~m}, 1 \mathrm{H}), 3.70(\mathrm{dd}, J=15.3,5.6 \mathrm{~Hz}, 1 \mathrm{H}), 3.63$ (dd, $J=15.5,6.0 \mathrm{~Hz}, 1 \mathrm{H}), 3.00(\mathrm{~s}, 3 \mathrm{H})$; ${ }^{13} \mathrm{C}$ NMR $(100 \mathrm{MHz}) \delta 172.4,153.9,147.2,132.0,122.4,113.9,109.3,81.8,54.8,39.6$; IR (neat) 1755 $(\mathrm{C}=\mathrm{O}) \mathrm{cm}^{-1}$; HRMS (EI) calcd for $\mathrm{C}_{12} \mathrm{H}_{13} \mathrm{BrNO}_{2} 282.0130$, found $282.0130(\mathrm{M}+\mathrm{H})$.
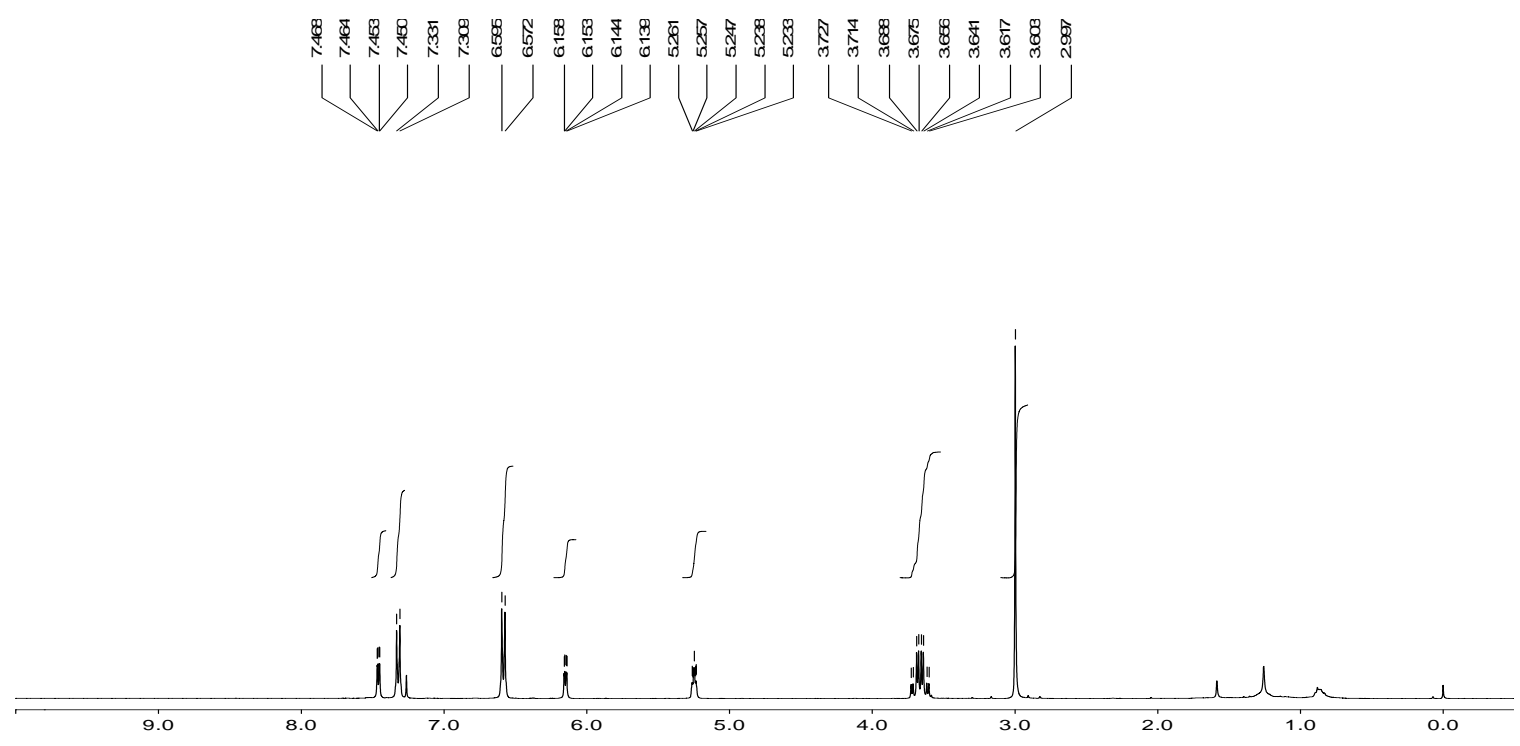
ppm (t1)
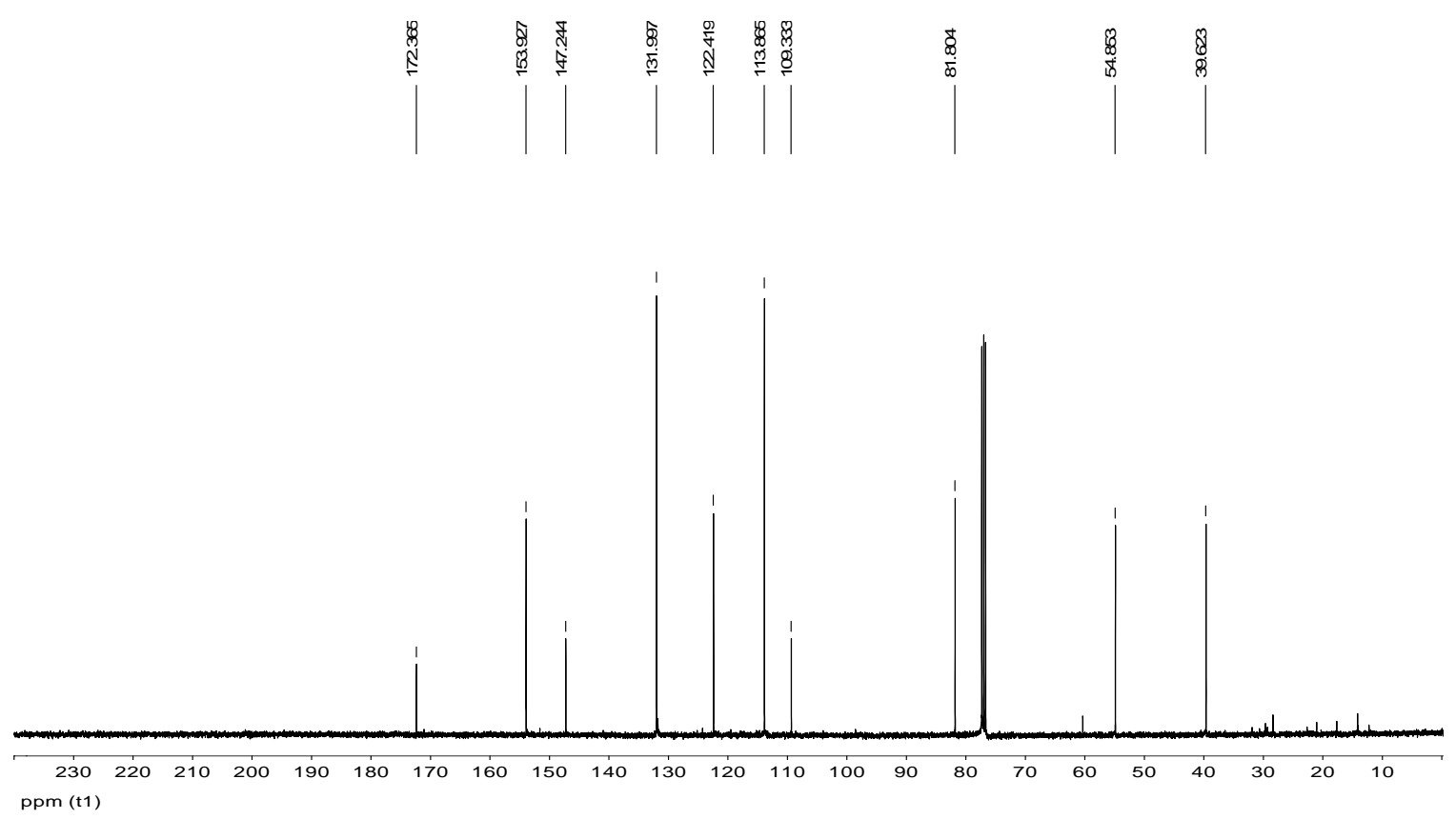


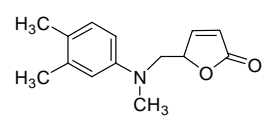

Table 2, entry 2: Purified by chromatography on silica gel $(5: 1 \rightarrow 1: 1$ hexanes/EtOAc); orange oil: $\mathrm{TLC} \mathrm{R}_{f}=0.14$ (3:1 hexanes/EtOAc); ${ }^{1} \mathrm{H} \mathrm{NMR}\left(400 \mathrm{MHz}, \mathrm{CDCl}_{3}\right) \delta 7.48$ (dd, $J=5.8,1.6 \mathrm{~Hz}, 1 \mathrm{H}), 7.00(\mathrm{~d}, J=8.1 \mathrm{~Hz}, 1 \mathrm{H}), 6.54-6.54(\mathrm{~m}, 1 \mathrm{H}), 6.50-6.47$ (m, 1H), 6.12 (dd, $J=5.8$, $1.6 \mathrm{~Hz}, 1 \mathrm{H}), 5.25$ (tt, $J=5.8,1.6 \mathrm{~Hz}, 1 \mathrm{H}), 3.69-3.58(\mathrm{~m}, 2 \mathrm{H}), 2.94(\mathrm{~s}, 3 \mathrm{H}), 2.24(\mathrm{~s}, 3 \mathrm{H}), 2.17(\mathrm{~s}, 3 \mathrm{H})$; ${ }^{13} \mathrm{C}$ NMR (100 MHz) $\delta 172.6,154.6,145.6,137.4,130.3,125.5,121.9,114.2,110.1,82.0,55.3,39.6,20.3$, 18.5; IR (neat) $1751(\mathrm{C}=\mathrm{O}) \mathrm{cm}^{-1}$; HRMS (EI) calcd for $\mathrm{C}_{14} \mathrm{H}_{18} \mathrm{NO}_{2} 232.1338$, found $232.1331(\mathrm{M}+\mathrm{H})$.
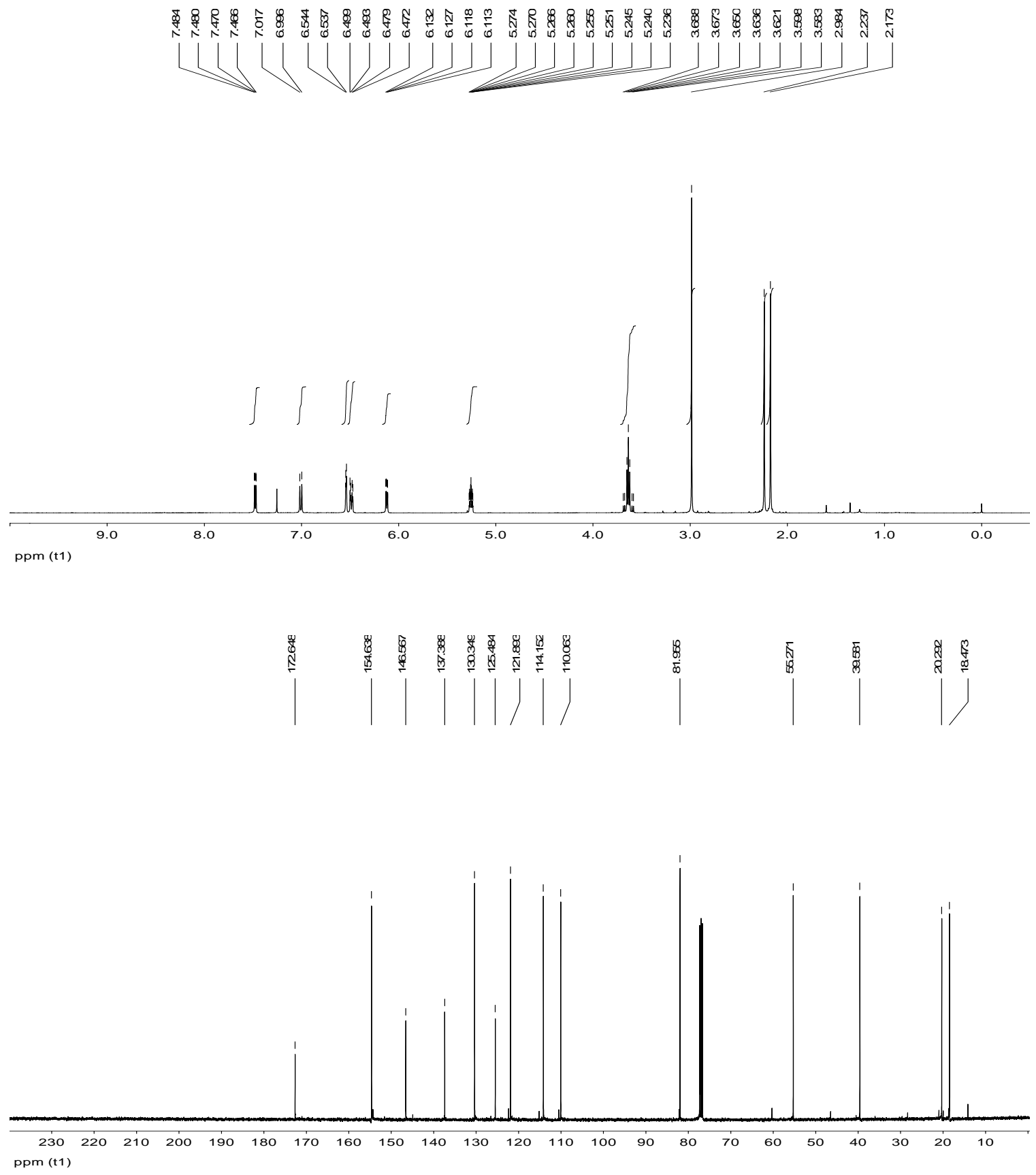


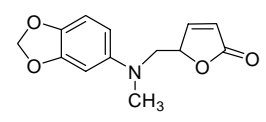

Table 2, entry 3: Purified by chromatography on silica gel $(5: 1 \rightarrow 1: 1$ hexanes/EtOAc); orange oil: $\mathrm{TLC} \mathrm{R}_{f}=0.36\left(1: 1\right.$ hexanes/EtOAc); ${ }^{1} \mathrm{H}$ NMR (400 MHz, $\left.\mathrm{CDCl}_{3}\right) \delta 7.47$ (dd, $J=5.6,1.6 \mathrm{~Hz}, 1 \mathrm{H}), 6.72(\mathrm{~d}, J=8.3 \mathrm{~Hz}, 1 \mathrm{H}), 6.38(\mathrm{~d}, J=2.6 \mathrm{~Hz}, 1 \mathrm{H}), 6.15(\mathrm{~d}, J=2.6 \mathrm{~Hz}, 1 \mathrm{H}), 6.15-6.13$ (m, 1H), 5.89 (s, 2H), $5.24(\mathrm{tt}, J=5.8 \mathrm{~Hz}, 1.6 \mathrm{~Hz}, 1 \mathrm{H}), 3.64-3.53(\mathrm{~m}, 2 \mathrm{H}), 2.95(\mathrm{~s}, 3 \mathrm{H}) ;{ }^{13} \mathrm{C}$ NMR (100 MHz) $\delta 172.6,154.4,148.6,144.6,139.8,122.1,108.5,105.1,100.8,96.2,81.9,56.3,40.3$; IR (neat) $1751(\mathrm{C}=\mathrm{O}) \mathrm{cm}^{-1}$; HRMS (EI) calcd for $\mathrm{C}_{13} \mathrm{H}_{14} \mathrm{NO}_{4} 248.0923$, found $248.0927(\mathrm{M}+\mathrm{H})$.

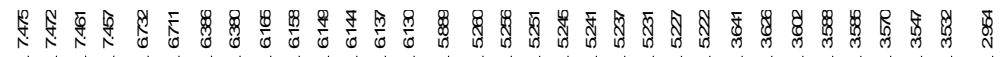

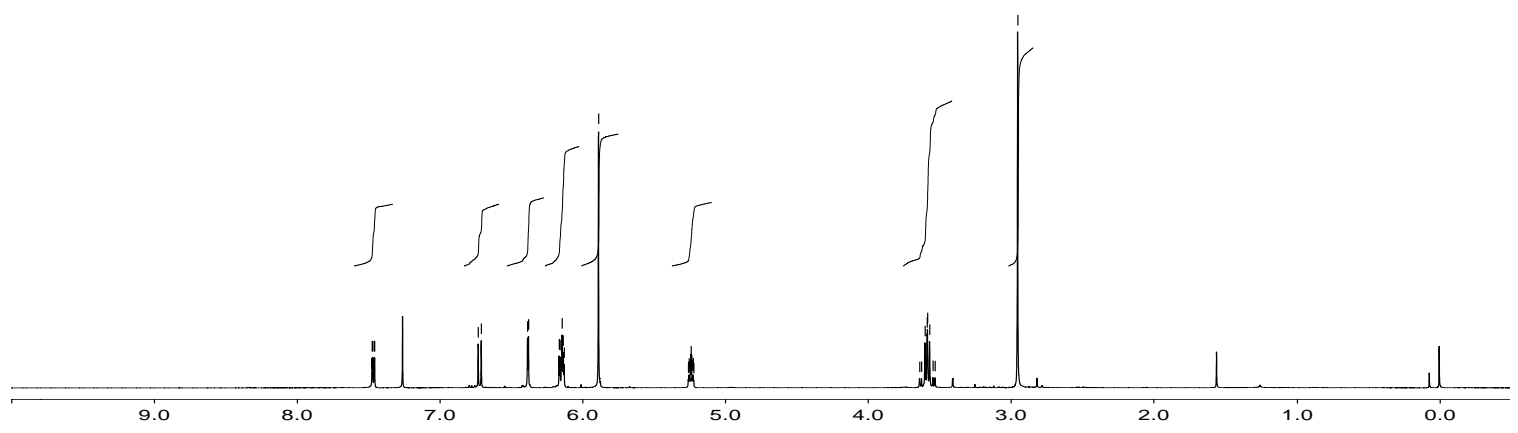
ppm (f1)
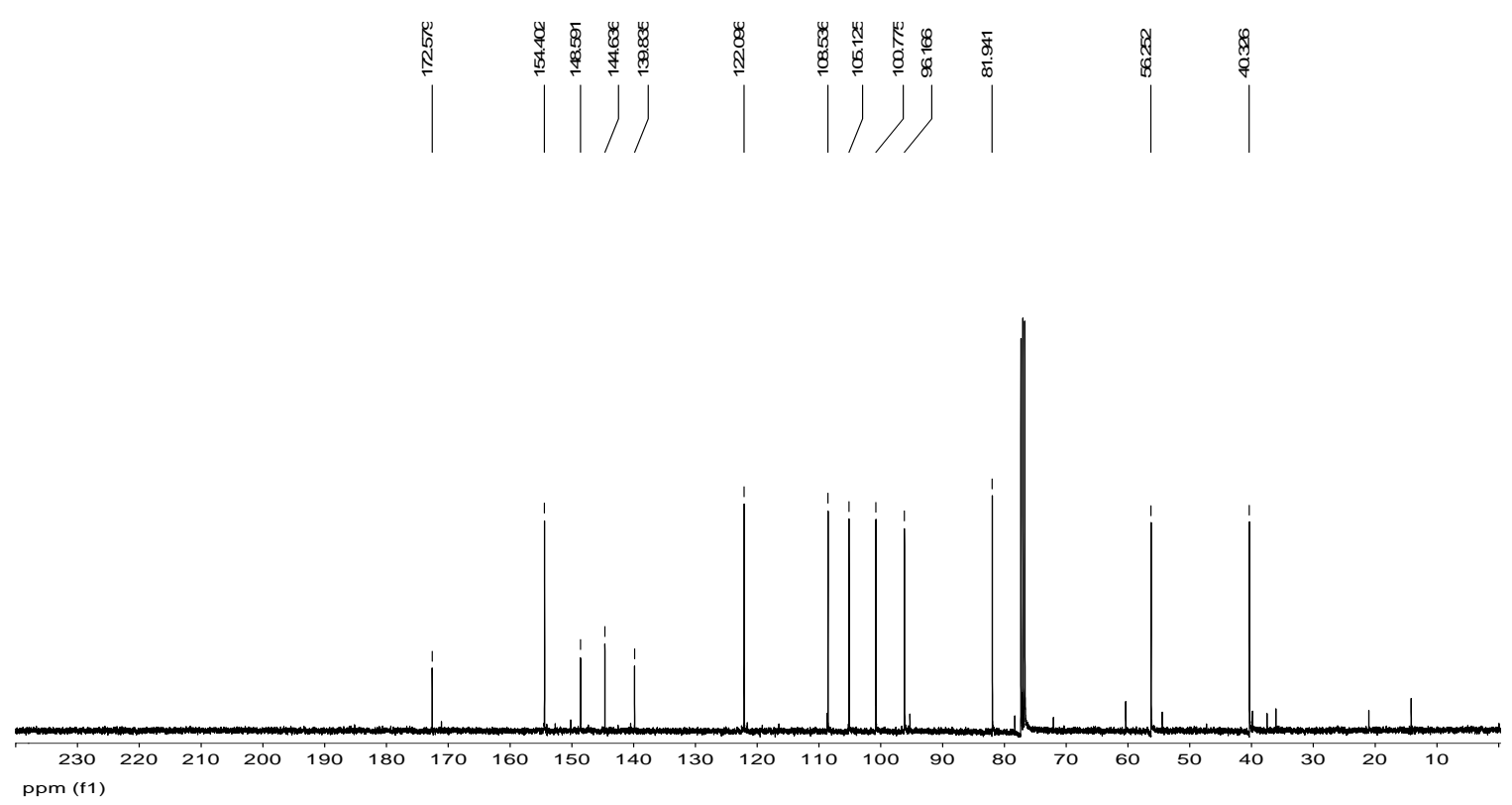


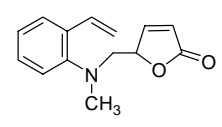

Table 2, entry 4: Purified by chromatography on silica gel $(8: 1 \rightarrow 2: 1$

hexanes/EtOAc); bright yellow oil: $\mathrm{TLC} \mathrm{R}_{f}=0.35$ (3:1 hexanes/EtOAc); ${ }^{1} \mathrm{H}$ NMR $\left(400 \mathrm{MHz}, \mathrm{CDCl}_{3}\right.$ ) $\delta 7.48(\mathrm{dd}, J=7.7,1.5 \mathrm{~Hz}, 1 \mathrm{H}), 7.34(\mathrm{dd}, J=5.7,1.6 \mathrm{~Hz}, 1 \mathrm{H}), 7.28-7.24(\mathrm{~m}, 1 \mathrm{H}), 7.14-7.01$ (comp, 3H), 6.07 (dd, $J=5.6,2.1 \mathrm{~Hz}, 1 \mathrm{H}) 5.68(\mathrm{dd}, J=17.7,1.4 \mathrm{~Hz}, 1 \mathrm{H}), 5.27$ (dd, $J=10.9,1.4 \mathrm{~Hz}, 1 \mathrm{H}), 5.11$ (tt, $J=5.6,1.6 \mathrm{~Hz}, 1 \mathrm{H}), 3.33(\mathrm{~d}, J=6.0 \mathrm{~Hz}, 2 \mathrm{H}), 2.86(\mathrm{~s}, 3 \mathrm{H}) ;{ }^{13} \mathrm{C}$ NMR $(100 \mathrm{MHz}) \delta 172.8,155.1,149.5$, 134.1, 133.0, 128.6, 127.0, 123.9, 121.7, 120.6, 114.3, 82.2, 58.4, 43.4; IR (neat) $1746(\mathrm{C}=\mathrm{O}) \mathrm{cm}^{-1}$; HRMS (EI) calcd for $\mathrm{C}_{14} \mathrm{H}_{16} \mathrm{NO}_{2} 230.1181$, found $230.1187(\mathrm{M}+\mathrm{H})$.

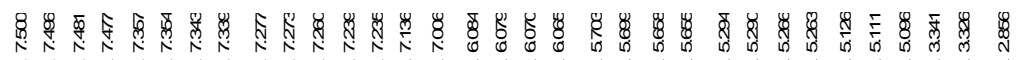

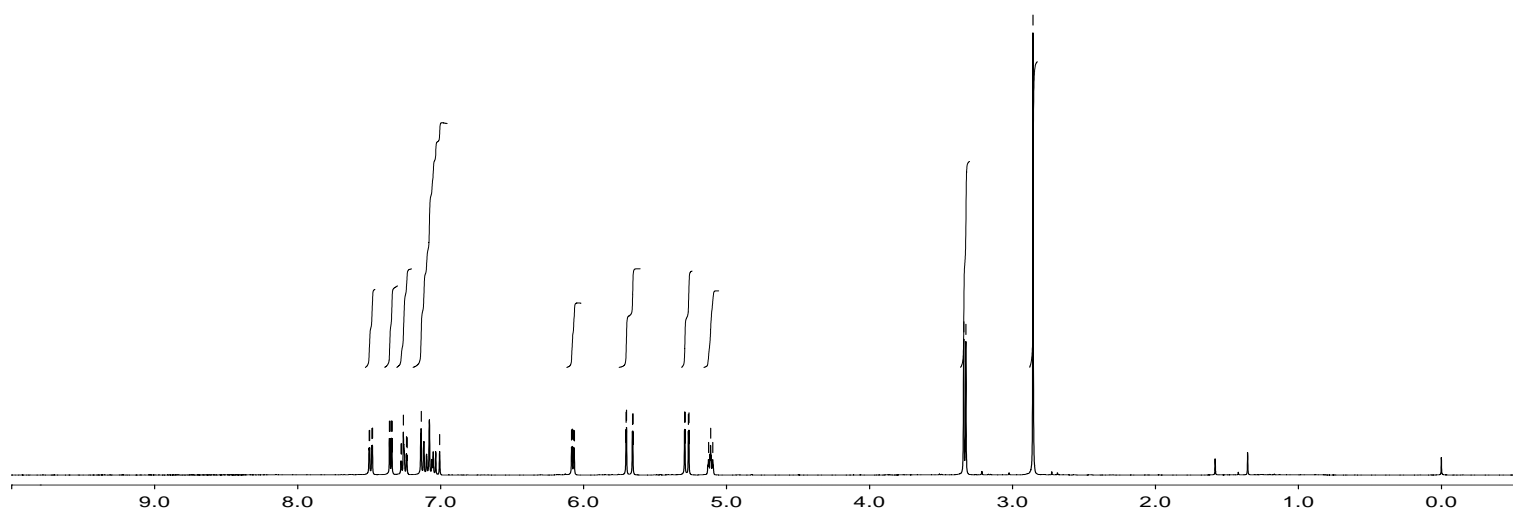
ppm (t1)
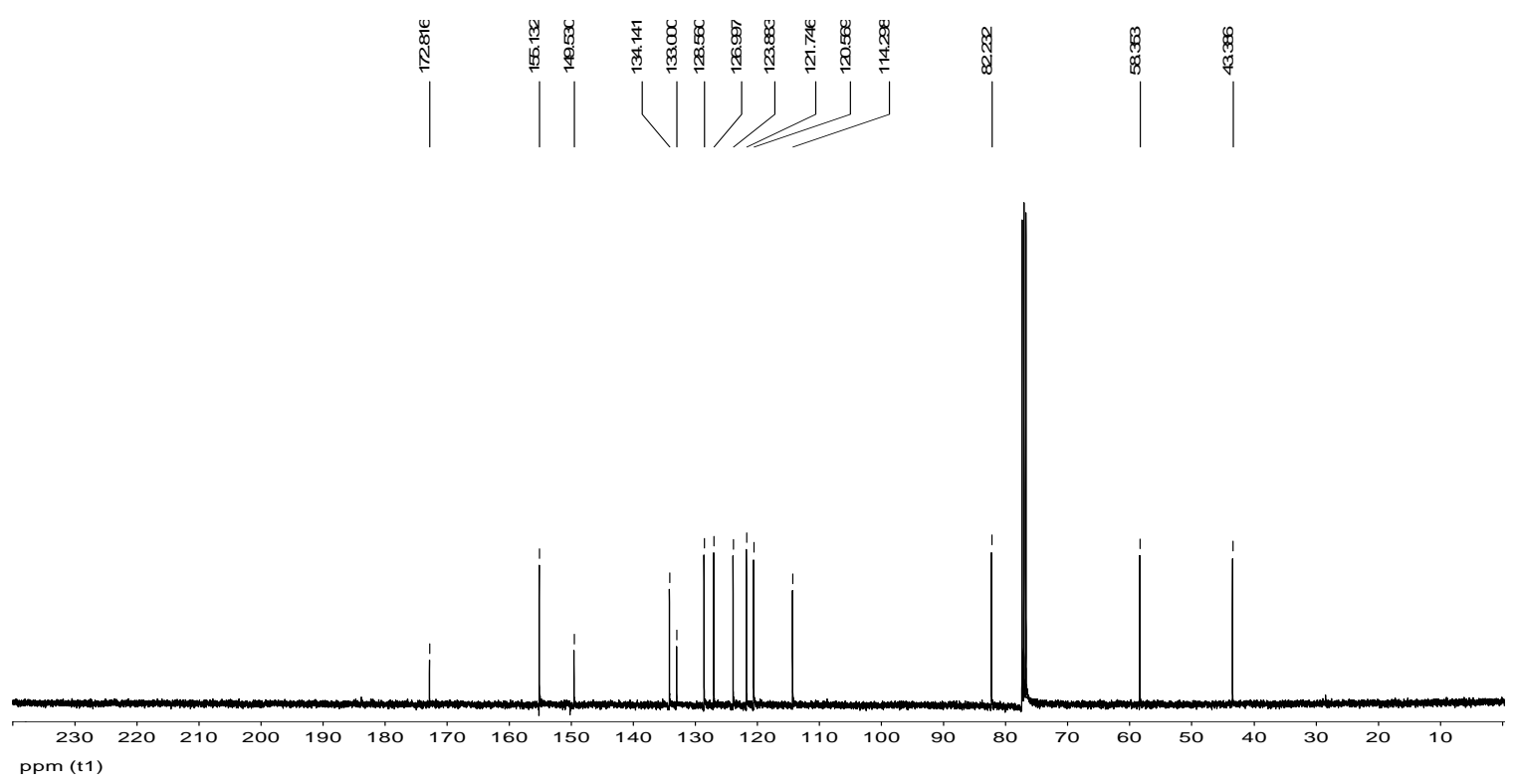


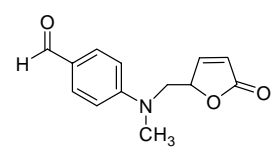

Table 2, entry 5: Purified by chromatography on silica gel $(2: 1 \rightarrow 1: 2$ hexanes/EtOAc); yellow oil: $\mathrm{TLC} \mathrm{R}_{f}=0.25$ (1:2 hexanes/EtOAc); ${ }^{1} \mathrm{H}$ NMR $\left(400 \mathrm{MHz}, \mathrm{CDCl}_{3}\right) \delta 9.78$ (s, $1 \mathrm{H}), 7.77(\mathrm{~d}, J=8.9 \mathrm{~Hz}, 2 \mathrm{H}), 7.50(\mathrm{dd}, J=5.8,1.4 \mathrm{~Hz}, 1 \mathrm{H}), 6.76(\mathrm{~d}, J=8.9 \mathrm{~Hz}, 2 \mathrm{H}), 6.19$ (dd, $J=5.5$, $2.0 \mathrm{~Hz}, 1 \mathrm{H}), 5.32-5.29(\mathrm{~m}, 1 \mathrm{H}), 3.89(\mathrm{dd}, J=15.5,5.0 \mathrm{~Hz}, 1 \mathrm{H}), 3.74(\mathrm{dd}, J=15.5,6.2 \mathrm{~Hz}, 1 \mathrm{H}), 3.14$ (s, $3 \mathrm{H}) ;{ }^{13} \mathrm{C}$ NMR (100 MHz) $\delta 190.2,172.1,153.4,152.7,132.0,126.1,122.7,111.2,81.7,54.1,39.8$; IR (neat) 1746, $1588(\mathrm{C}=\mathrm{O}) \mathrm{cm}^{-1}$; HRMS (EI) calcd for $\mathrm{C}_{13} \mathrm{H}_{14} \mathrm{NO}_{3} 232.0974$, found $232.0974(\mathrm{M}+\mathrm{H})$.
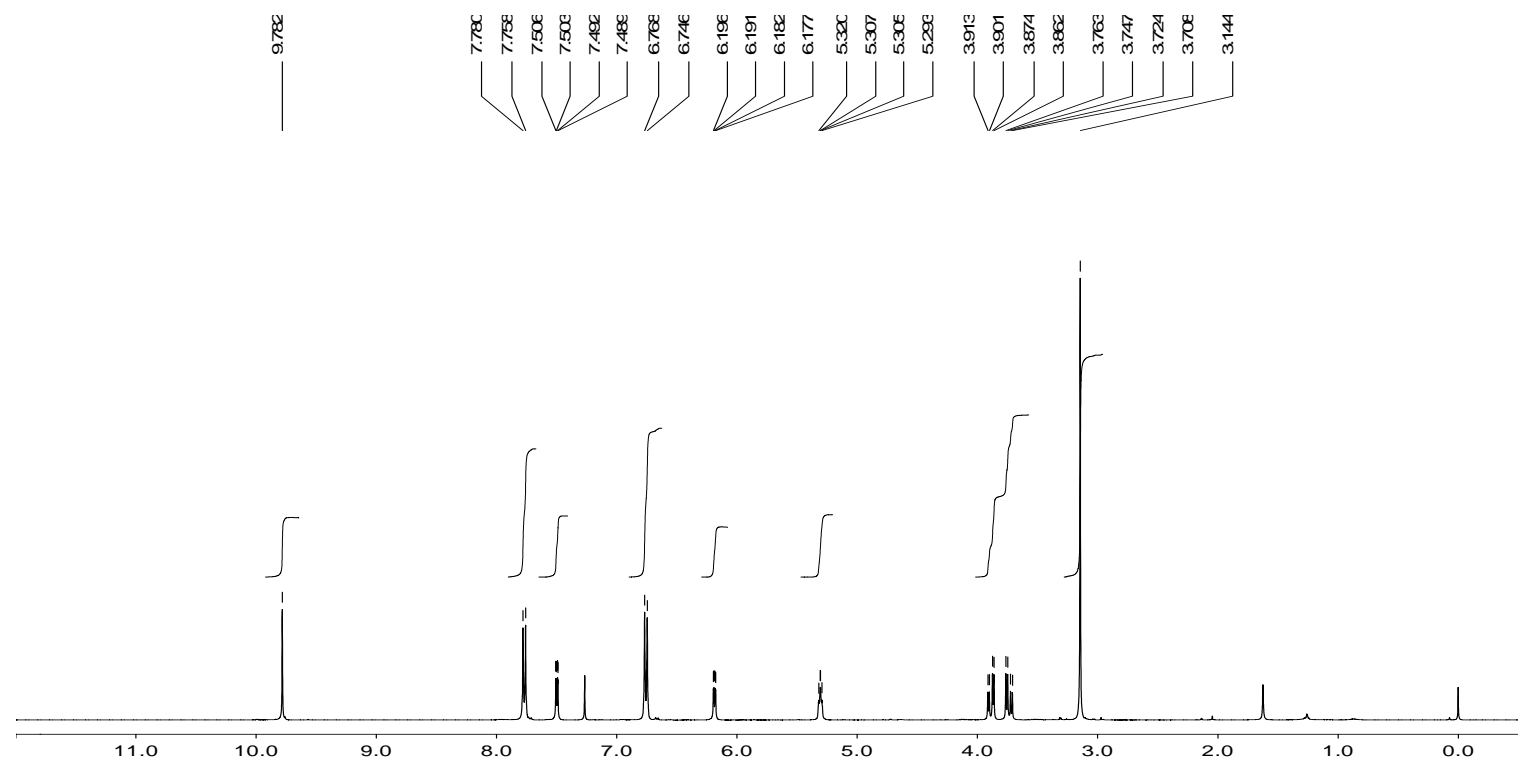

ppm (t1)

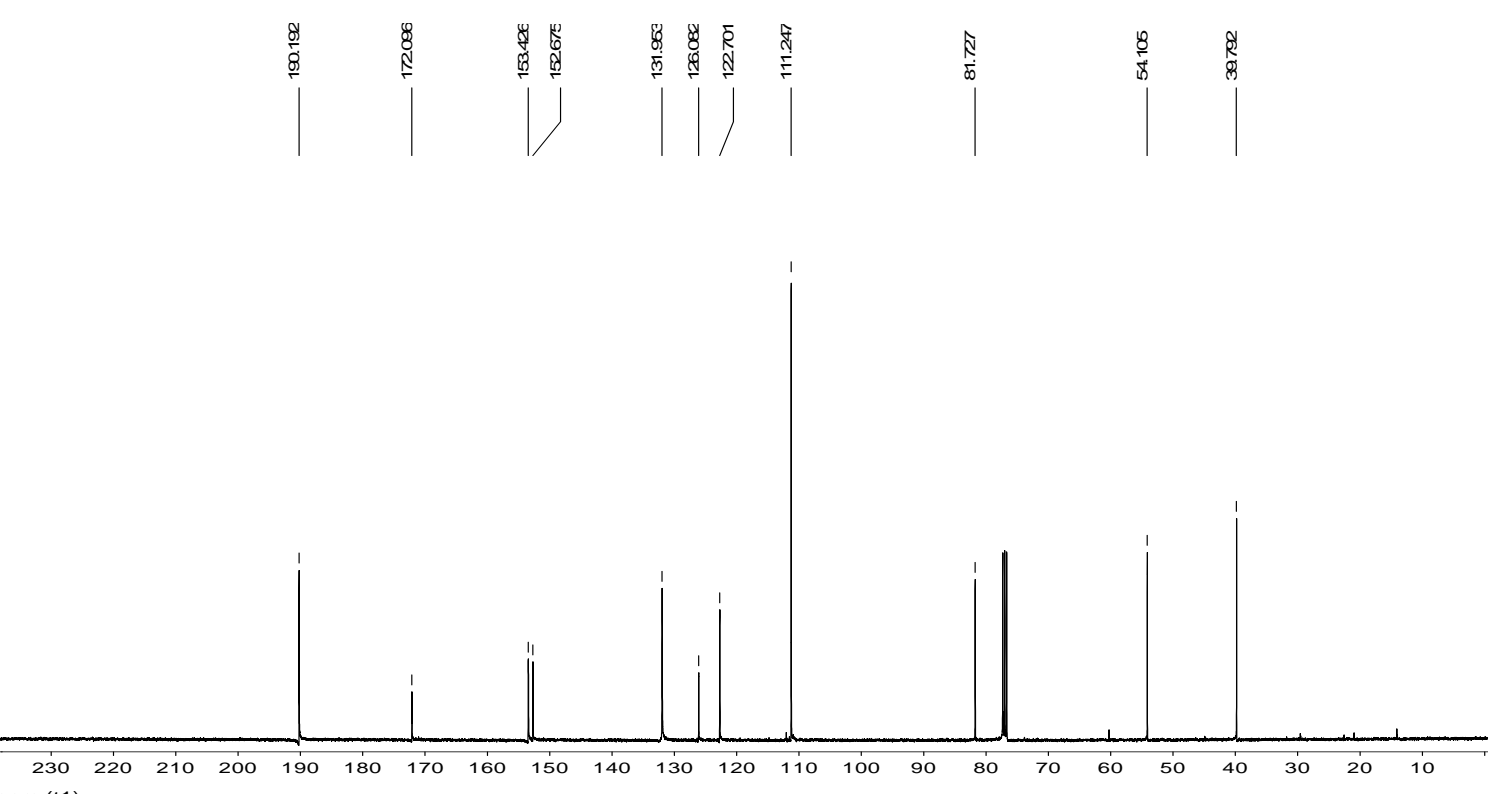
ppm (t1) 
Table 2, entry 6: Purified by chromatography on silica gel $(5: 1 \rightarrow 2: 1$ hexanes/EtOAc); yellow oil: $\mathrm{TLC} \mathrm{R}_{f}=0.22$ (3:1 hexanes/EtOAc); ${ }^{1} \mathrm{H}$ NMR $\left(400 \mathrm{MHz}, \mathrm{CDCl}_{3}\right) \delta 7.50(\mathrm{~d}$, $J=5.8 \mathrm{~Hz}, 1 \mathrm{H}), 7.27-7.23(\mathrm{~m}, 2 \mathrm{H}), 6.73-6.70$ (comp, 3H), $6.16-6.14(\mathrm{~m}, 1 \mathrm{H}), 5.27-5.34(\mathrm{~m}, 1 \mathrm{H})$, $3.70(\mathrm{dd}, J=15.2,5.8 \mathrm{~Hz}, 1 \mathrm{H}), 3.57$ (dd, $J=15.3,6.4 \mathrm{~Hz}, 1 \mathrm{H}), 3.52-3.34(\mathrm{~m}, 2 \mathrm{H}), 1.17$ (t, $J=7.0 \mathrm{~Hz}$, $3 \mathrm{H}) ;{ }^{13} \mathrm{C}$ NMR (100 MHz) $\delta 172.6,154.7,146.9,129.5,122.1,117.1,112.5,81.8,52.9,45.8,11.9$; IR (neat) $1751(\mathrm{C}=\mathrm{O}) \mathrm{cm}^{-1}$; HRMS (EI) calcd for $\mathrm{C}_{13} \mathrm{H}_{16} \mathrm{NO}_{2} 218.1181$, found $218.1184(\mathrm{M}+\mathrm{H})$.

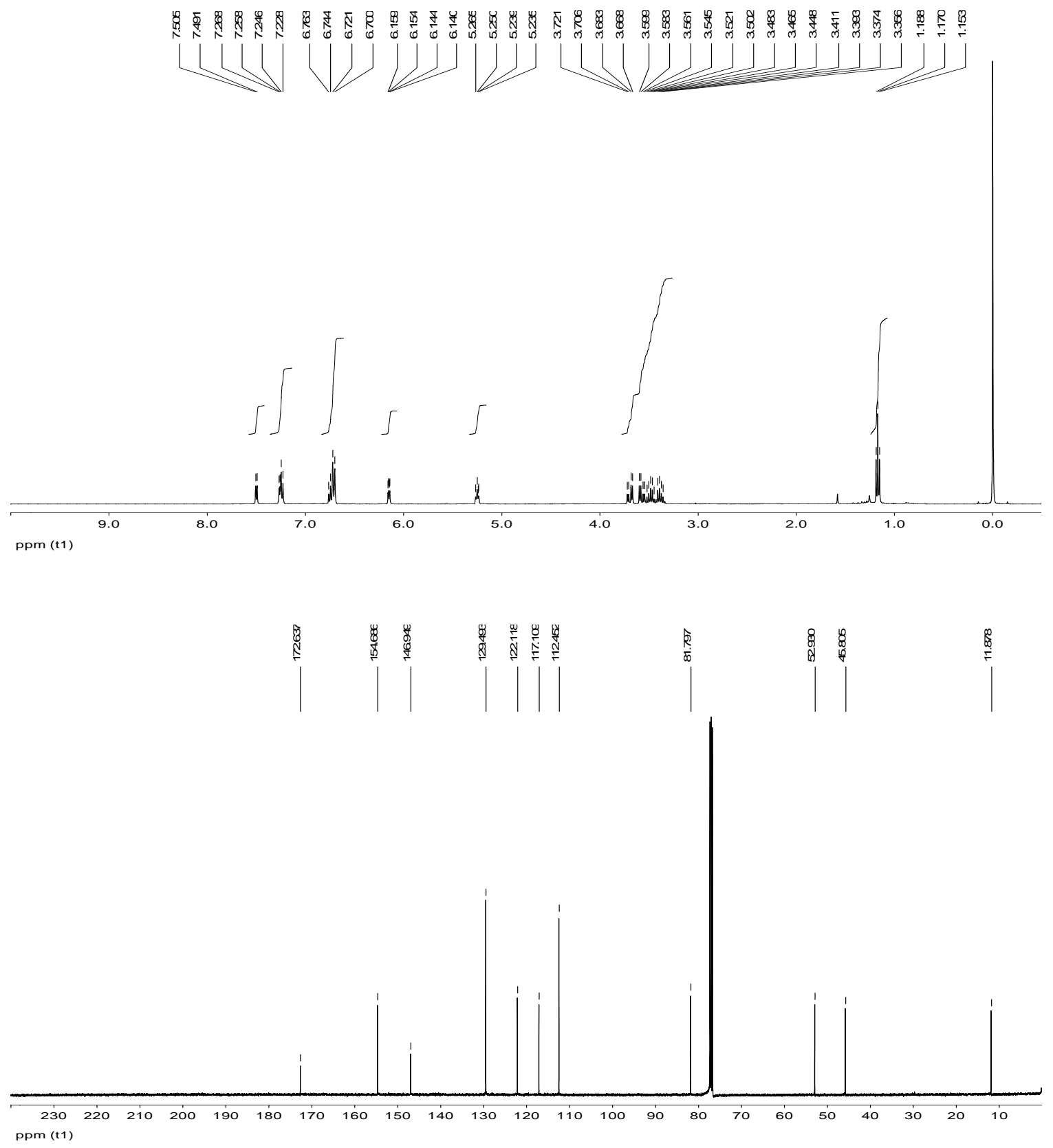


Table 2, entry 7: Purified by chromatography on silica gel $(6: 1 \rightarrow 3: 1$ hexanes/EtOAc); yellow oil: $\mathrm{TLC} \mathrm{R}_{f}=0.26$ (3:1 hexanes/EtOAc); ${ }^{1} \mathrm{H}$ NMR (400 MHz, $\left.\mathrm{CDCl}_{3}\right) \delta 7.42$ (dd, $J=5.6,1.4 \mathrm{~Hz}, 1 \mathrm{H}), 7.30-7.17(\mathrm{comp}, 7 \mathrm{H}), 6.73(\mathrm{t}, J=7.2 \mathrm{~Hz}, 1 \mathrm{H}), 6.63(\mathrm{~d}, J=8.1 \mathrm{~Hz}, 2 \mathrm{H}), 6.10$ (dd, $J$ $=5.8,1.9 \mathrm{~Hz}, 1 \mathrm{H}), 5.21-5.18(\mathrm{~m}, 1 \mathrm{H}), 3.65(\mathrm{dd}, J=15.2,6.0 \mathrm{~Hz}, 1 \mathrm{H}), 3.56(\mathrm{dd}, J=15.2,6.2 \mathrm{~Hz}, 1 \mathrm{H})$, 3.44 - 3.27 (m, 2H), 2.64 (t, $J=7.6 \mathrm{~Hz}, 2 \mathrm{H}), 1.92$ (p, $J=7.6 \mathrm{~Hz}, 2 \mathrm{H}) ;{ }^{13} \mathrm{C}$ NMR $(100 \mathrm{MHz}) \delta 172.5,154.5$, 146.9, 141.2, 129.4, 128.4, 128.2, 126.0, 122.0, 117.2, 112.7, 81.6, 53.5, 51.0, 33.0, 28.1; IR (neat) 1765 $(\mathrm{C}=\mathrm{O}) \mathrm{cm}^{-1}$; HRMS (EI) calcd for $\mathrm{C}_{20} \mathrm{H}_{22} \mathrm{NO}_{2} 308.1651$, found $308.1643(\mathrm{M}+\mathrm{H})$.
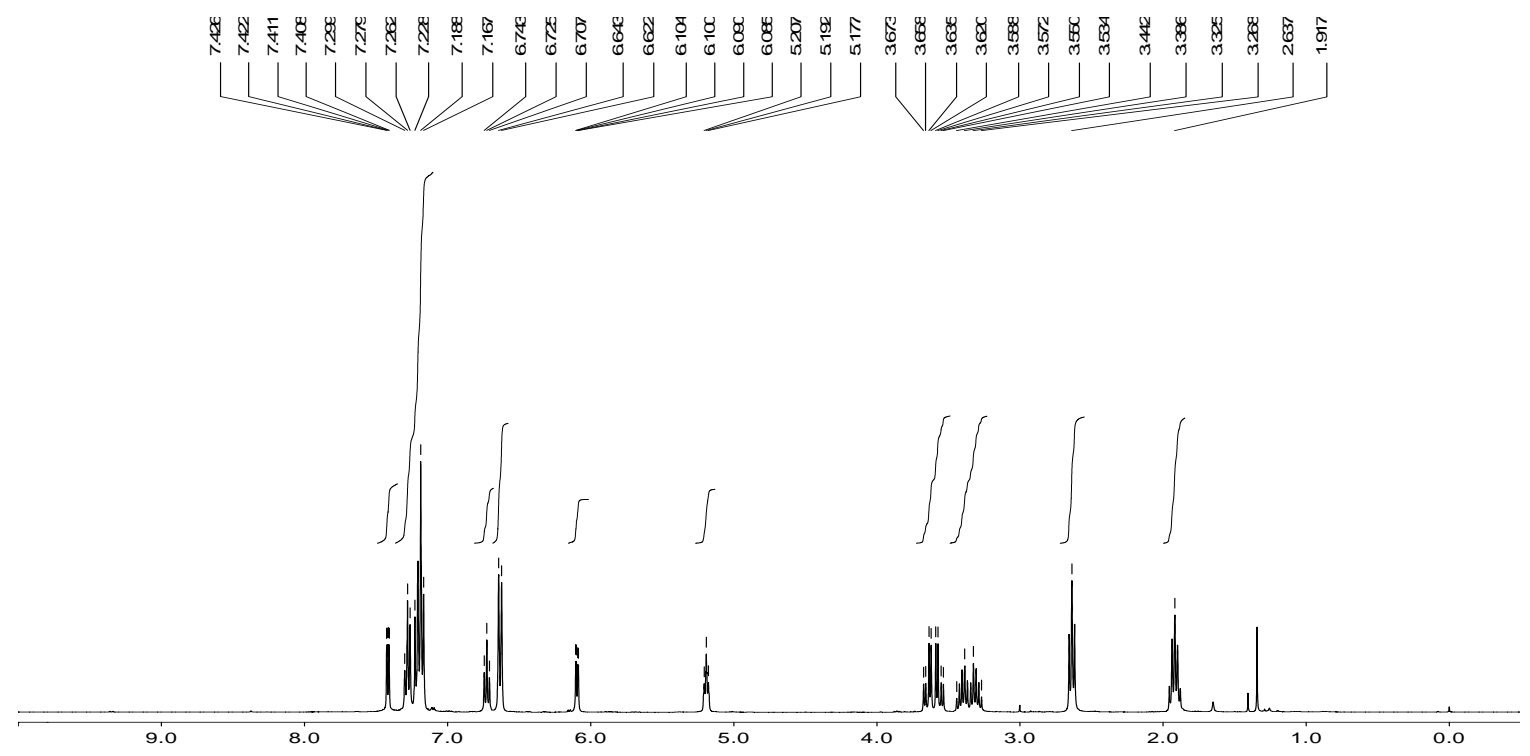
ppm (t1)
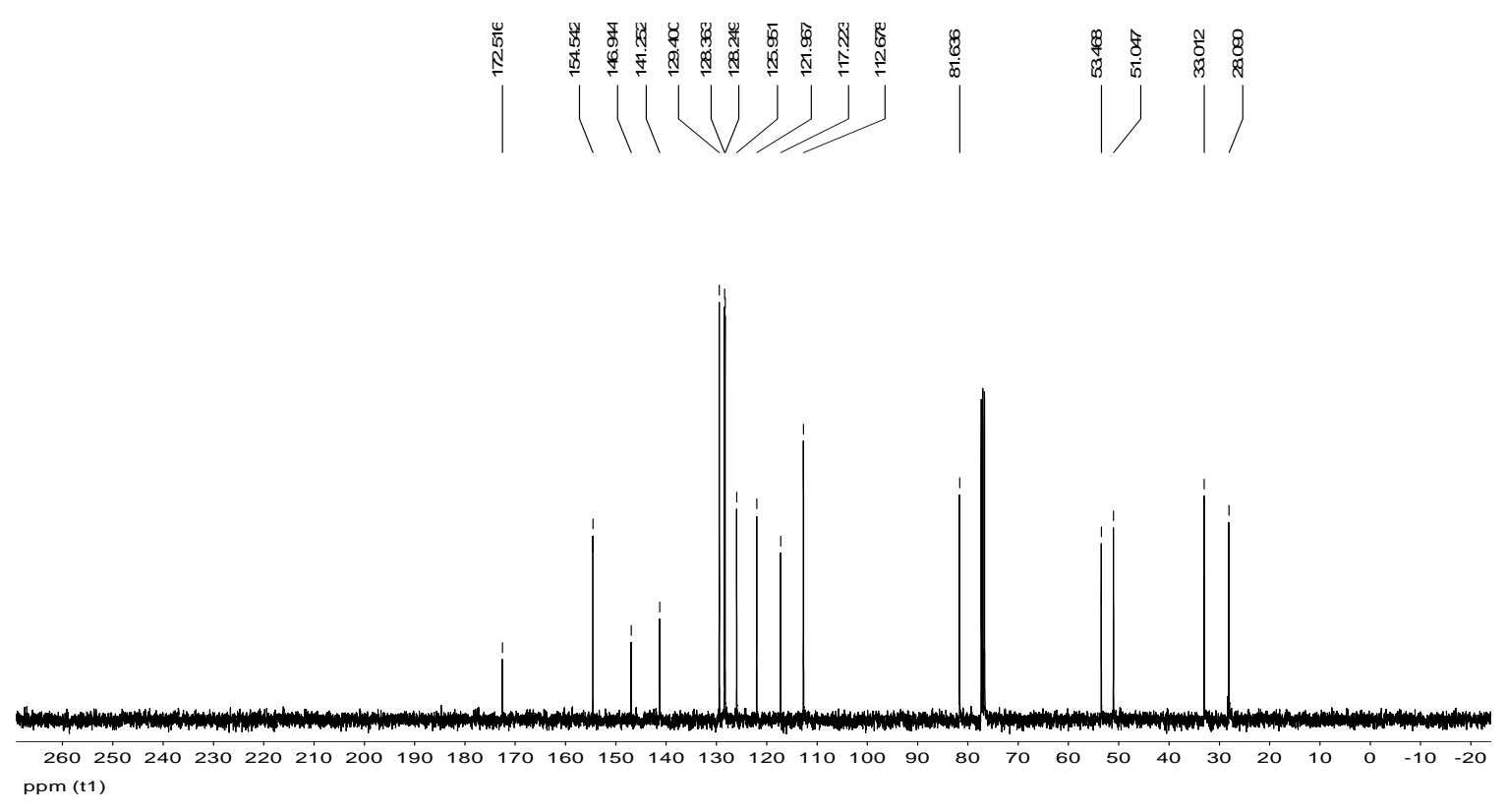


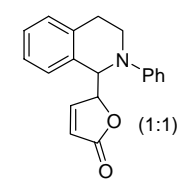

Table 2, entry 8: Purified by chromatography on silica gel $(5: 1 \rightarrow 1: 1$ hexanes/EtOAc); orange oil: $\mathrm{TLC} \mathrm{R}_{f}=0.26$ (3:1 hexanes/EtOAc); ${ }^{1} \mathrm{H}$ NMR $\left(400 \mathrm{MHz}, \mathrm{CDCl}_{3}\right) \delta 7.52(\mathrm{~d}$, $J=5.8 \mathrm{~Hz}, 1 \mathrm{H}), 7.38$ (d, $J=5.8 \mathrm{~Hz}, 1 \mathrm{H}), 7.33-7.18$ (comp, 6H), 7.00 (d, $J=8.3 \mathrm{~Hz}, 1 \mathrm{H}), 6.89$ (d, $J=$ $8.3 \mathrm{~Hz}, 1 \mathrm{H}), 6.86-6.80(\mathrm{~m}, 1 \mathrm{H}), 6.14-6.12(\mathrm{~m}, 1 \mathrm{H}), 5.94-5.92(\mathrm{~m}, 1 \mathrm{H}), 5.46-5.45(\mathrm{~m}, 1 \mathrm{H}), 5.36-$ $5.34(\mathrm{~m}, 1 \mathrm{H}), 5.18(\mathrm{~d}, J=4.2 \mathrm{~Hz}, 1 \mathrm{H}), 4.91(\mathrm{~d}, J=6.2 \mathrm{~Hz}, 1 \mathrm{H}), 3.82-3.76(\mathrm{~m}, 1 \mathrm{H}), 3.67-3.55$ (comp, 2H), $3.47-3.41(\mathrm{~m}, 1 \mathrm{H}), 3.10-2.91(\mathrm{comp}, 4 \mathrm{H}) ;{ }^{13} \mathrm{C} \mathrm{NMR}(100 \mathrm{MHz}) \delta 172.5,172.4,154.6,153.6$, $148.9,148.8,135.7,135.3,132.4,131.9,129.4,129.4,128.6,128.4,128.1,127.8,127.7,127.5,126.3$, $125.9,122.5,122.2,118.8,118.6,114.5,114.4,85.9,85.4,61.7,60.5,44.0,43.4,28.3,27.2$; IR (neat) 1756 $(\mathrm{C}=\mathrm{O}) \mathrm{cm}^{-1}$; HRMS (EI) calcd for $\mathrm{C}_{19} \mathrm{H}_{18} \mathrm{NO}_{2} 292.1338$, found $292.1331(\mathrm{M}+\mathrm{H})$.

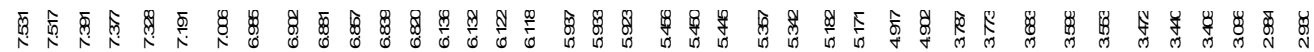

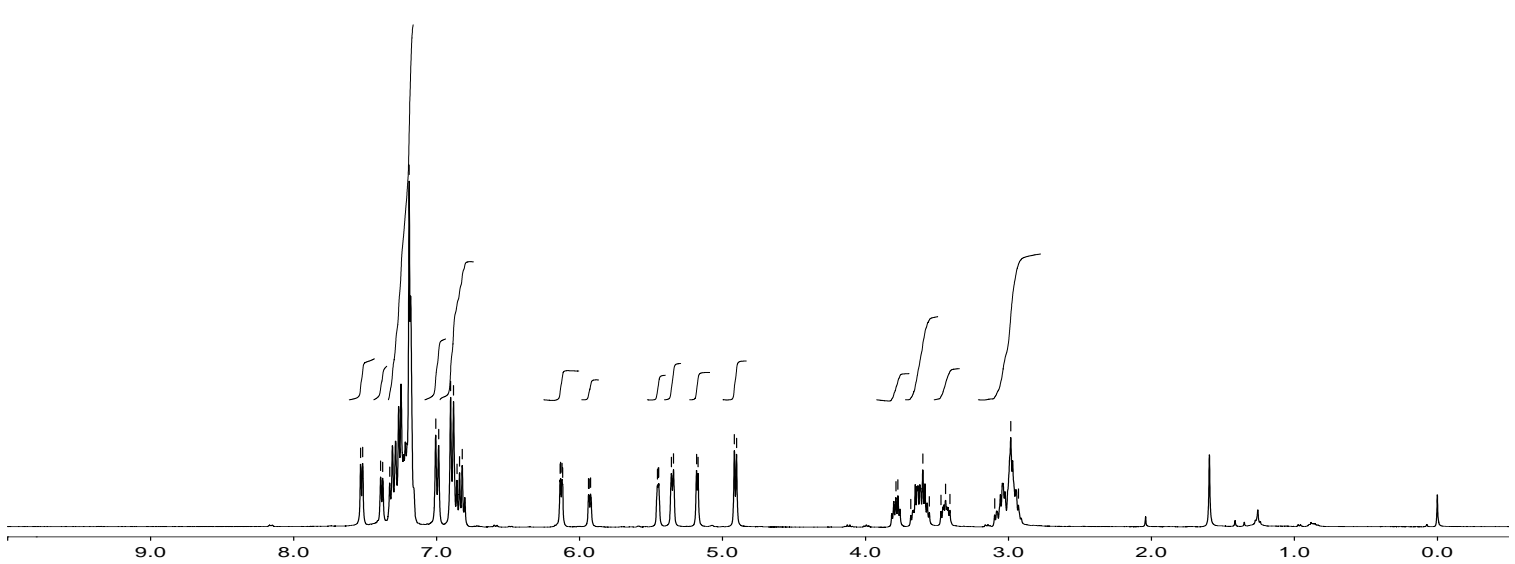

ppm (t1)
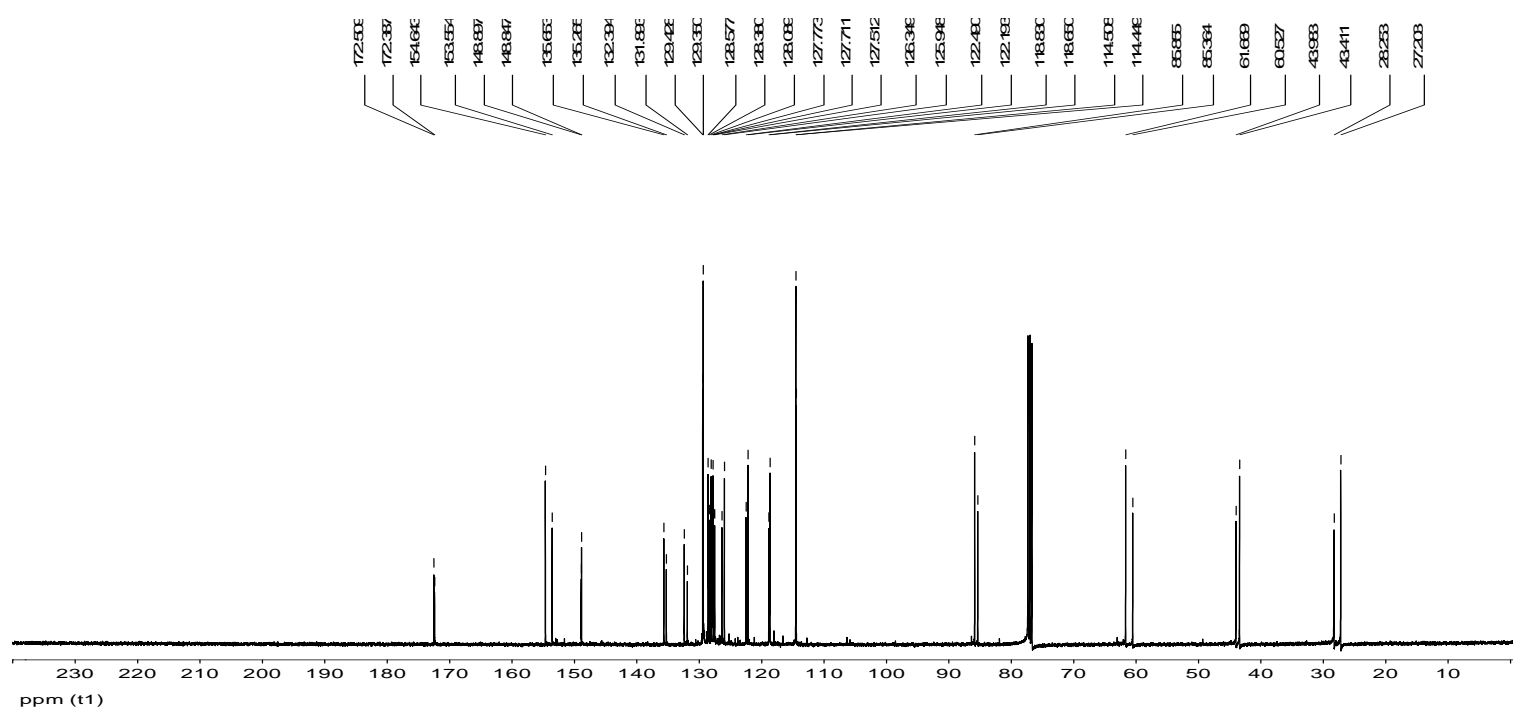


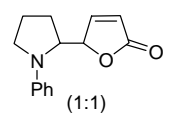

Table 2, entry 9: Purified by chromatography on silica gel $(5: 1 \rightarrow 1: 1$ hexanes/EtOAc); yellow oil: $\mathrm{TLC} \mathrm{R}_{f}=0.22\left(3: 1\right.$ hexanes/EtOAc); ${ }^{1} \mathrm{H} \mathrm{NMR}\left(400 \mathrm{MHz}, \mathrm{CDCl}_{3}\right) \delta 7.46-$ 7.40 (comp, 2H), $7.30-7.22$ (comp, 4H), $6.80-6.72$ (comp, 4H), 6.59 (d, J = 8.1 Hz, 2H), $6.19-6.16$ (comp, 2H), $5.40-5.39$ (m, 1H), $5.03-5.02(\mathrm{~m}, 1 \mathrm{H}), 4.40-4.37$ (m, 1H), $3.86(\mathrm{t}, J=7.2 \mathrm{~Hz}, 1 \mathrm{H}), 3.64-$ 3.55 (comp, 2H), 3.24 - 3.16 (comp, 2H), $2.17-1.68$ (comp, 8H); ${ }^{13} \mathrm{C}$ NMR (100 MHz) $\delta 172.8,172.7$, 155.9, 153.6, 1467.0, 146.8, 129.4, 129.2, 122.7, 121.6, 117.2, 117.0, 112.6, 112.4, 84.0, 82.4, 60.5, 59.1, 49.4, 49.1, 27.8, 25.2, 24.1, 23.2; IR (neat) $1756(\mathrm{C}=\mathrm{O}) \mathrm{cm}^{-1}$; HRMS (EI) calcd for $\mathrm{C}_{14} \mathrm{H}_{16} \mathrm{NO}_{2} 230.1181$, found $230.1177(\mathrm{M}+\mathrm{H})$.

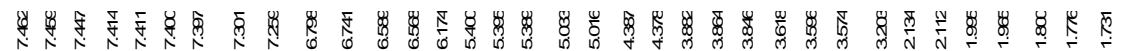

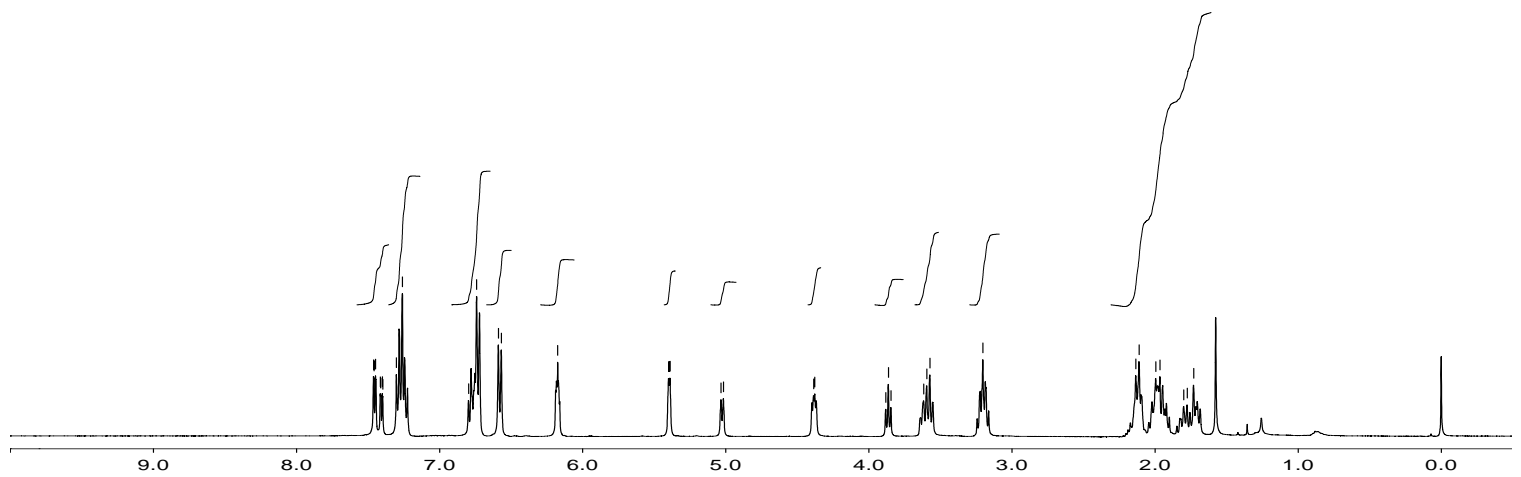
ppm (t1)
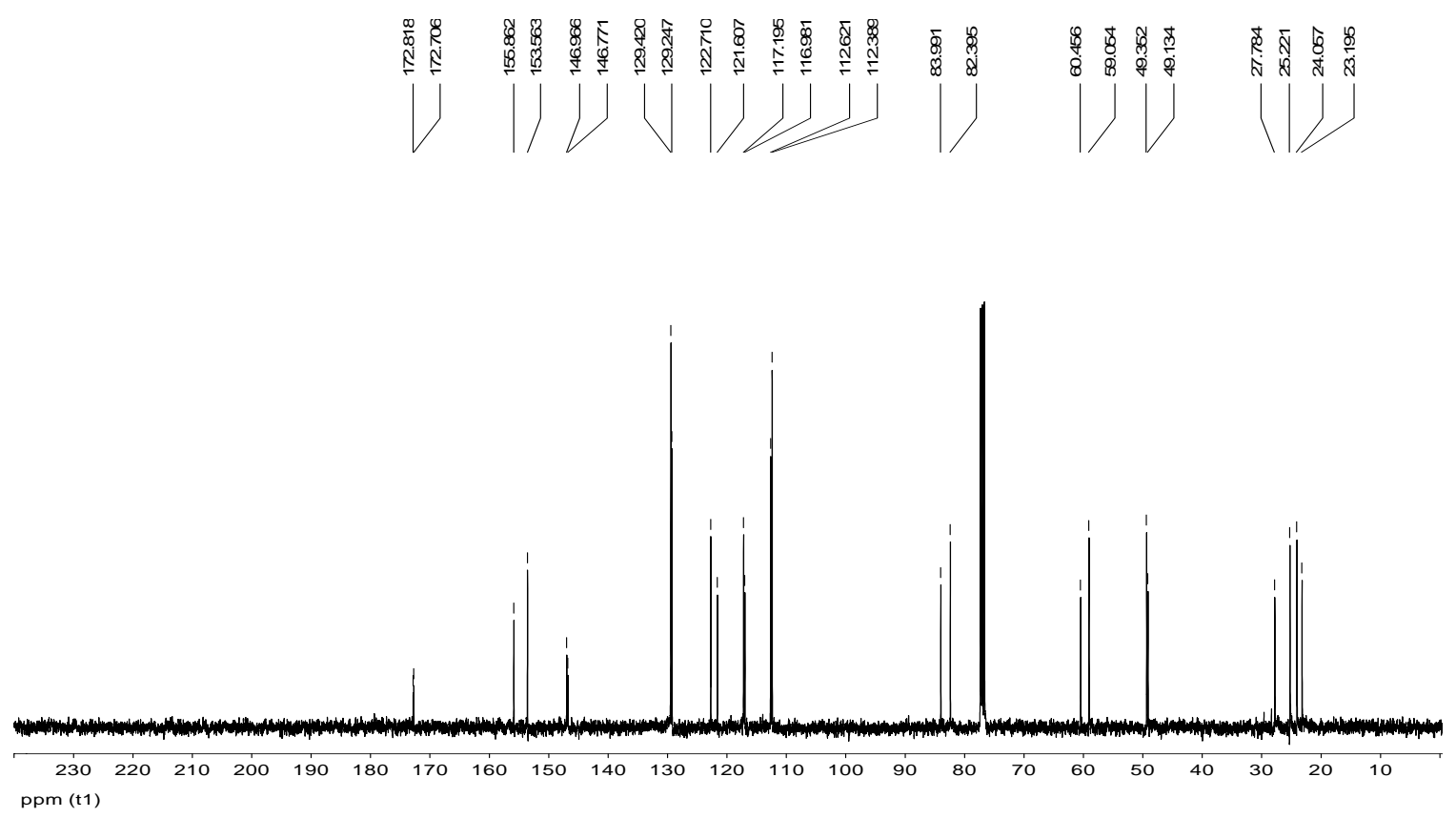


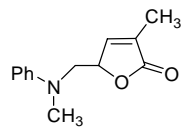

Table 2, entry 10: Purified by chromatography on silica gel $(5: 1 \rightarrow 1: 1$ hexanes/EtOAc); yellow oil: $\mathrm{TLC} \mathrm{R}_{f}=0.27$ (3:1 hexanes/EtOAc); ${ }^{1} \mathrm{H} \mathrm{NMR}\left(400 \mathrm{MHz}, \mathrm{CDCl}_{3}\right) \delta 7.26-$ $7.22(\mathrm{~m}, 2 \mathrm{H}), 7.05-7.04$ (m, 1H), $6.76-6.70$ (comp, 3H), $5.12-5.09$ (m, 1H), 3.65 (dd, J = 15.3, $5.3 \mathrm{~Hz}$, $1 \mathrm{H}), 3.55(\mathrm{dd}, J=15.3,5.9 \mathrm{~Hz}, 1 \mathrm{H}), 3.00(\mathrm{~s}, 3 \mathrm{H}), 1.89$ (s, 3H); ${ }^{13} \mathrm{C}$ NMR $(100 \mathrm{MHz}) \delta 173.7,148.3,146.7$, 130.6, 129.2, 117.1, 112.2, 79.6, 55.3, 39.4, 10.6; IR (neat) $1751(\mathrm{C}=\mathrm{O}) \mathrm{cm}^{-1}$; HRMS (EI) calcd for $\mathrm{C}_{13} \mathrm{H}_{16} \mathrm{NO}_{2} 218.1181$, found 218.1181 (M+H).
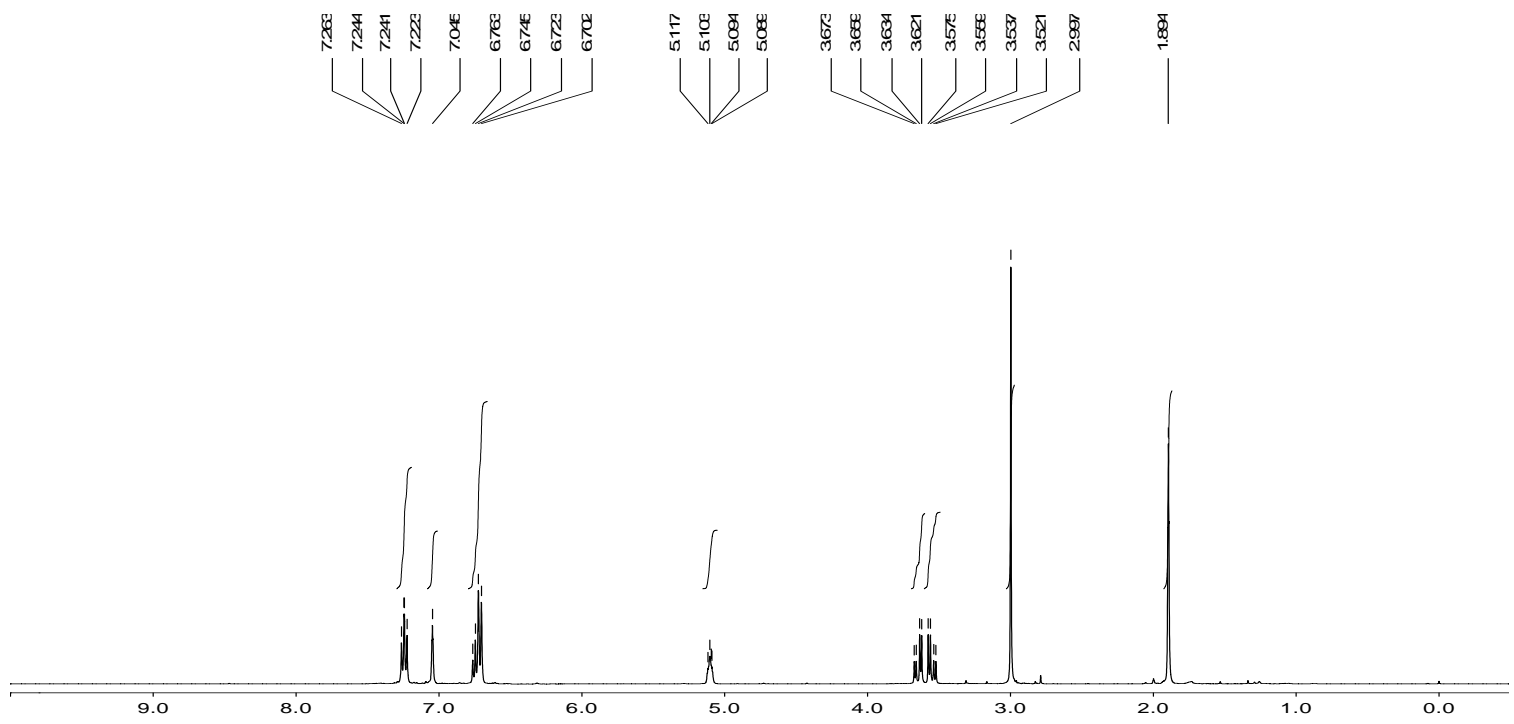
ppm (t1)
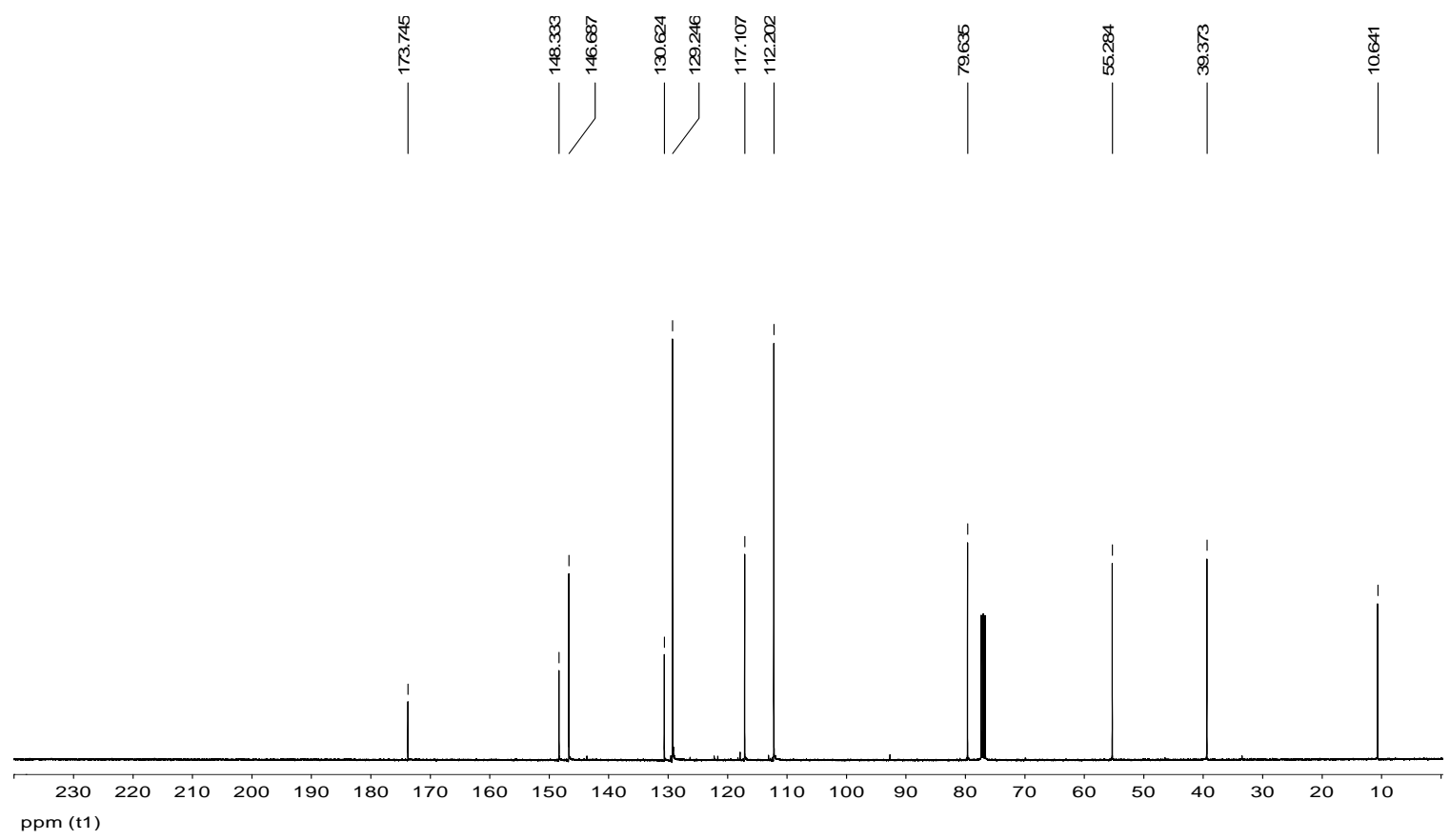


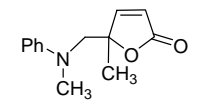

Table 2, entry 11: Purified by chromatography on silica gel $(5: 1 \rightarrow 1: 1$ hexanes/EtOAc); light yellow solid $\left(\mathrm{mp}=116-117^{\circ} \mathrm{C}\right): \quad \mathrm{TLC}_{f}=0.44\left(3: 1\right.$ hexanes/EtOAc); ${ }^{1} \mathrm{H}$ NMR $\left(400 \mathrm{MHz}, \mathrm{CDCl}_{3}\right) \delta 7.36(\mathrm{~d}, J=5.6 \mathrm{~Hz}, 1 \mathrm{H}), 7.23-7.19(\mathrm{~m}, 2 \mathrm{H}), 6.72(\mathrm{t}, J=7.2 \mathrm{~Hz}, 1 \mathrm{H}), 6.65(\mathrm{~d}, J=$ $8.1 \mathrm{~Hz}, 2 \mathrm{H}), 5.92$ (d, $J=5.6 \mathrm{~Hz}, 1 \mathrm{H}), 3.69$ (d, $J=15.5 \mathrm{~Hz}, 1 \mathrm{H}), 3.63$ (d, $J=15.7 \mathrm{~Hz}, 1 \mathrm{H}), 2.97$ (s, 3H), 1.51 (s, 3H); ${ }^{13} \mathrm{C}$ NMR (100 MHz) $\delta 171.9,157.8,148.8,129.1,121.2,117.0,112.0,90.5,58.8,40.0,21.8$; IR (neat) $1751(\mathrm{C}=\mathrm{O}) \mathrm{cm}^{-1}$; HRMS (EI) calcd for $\mathrm{C}_{13} \mathrm{H}_{16} \mathrm{NO}_{2} 218.1181$, found $218.1183(\mathrm{M}+\mathrm{H})$.
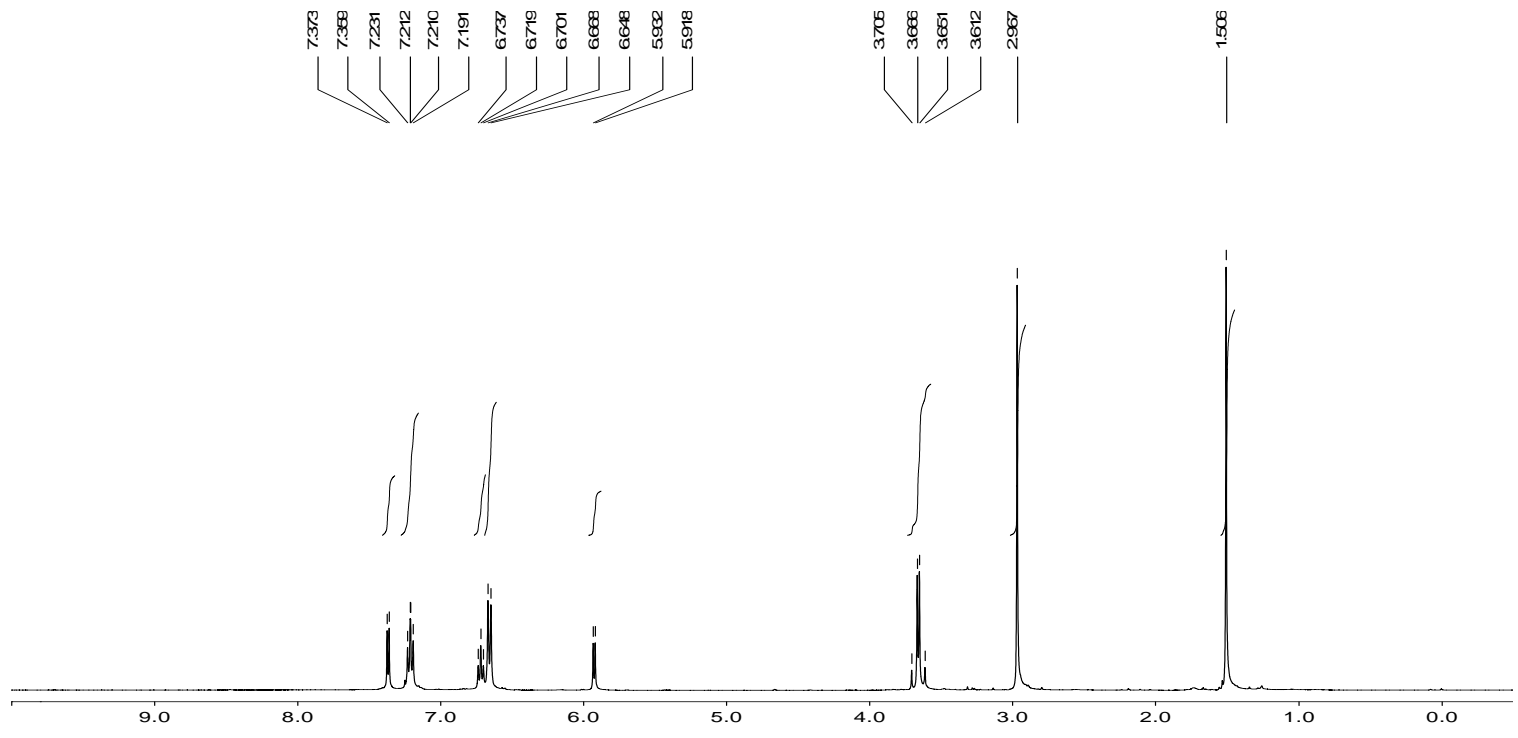
ppm (t1)

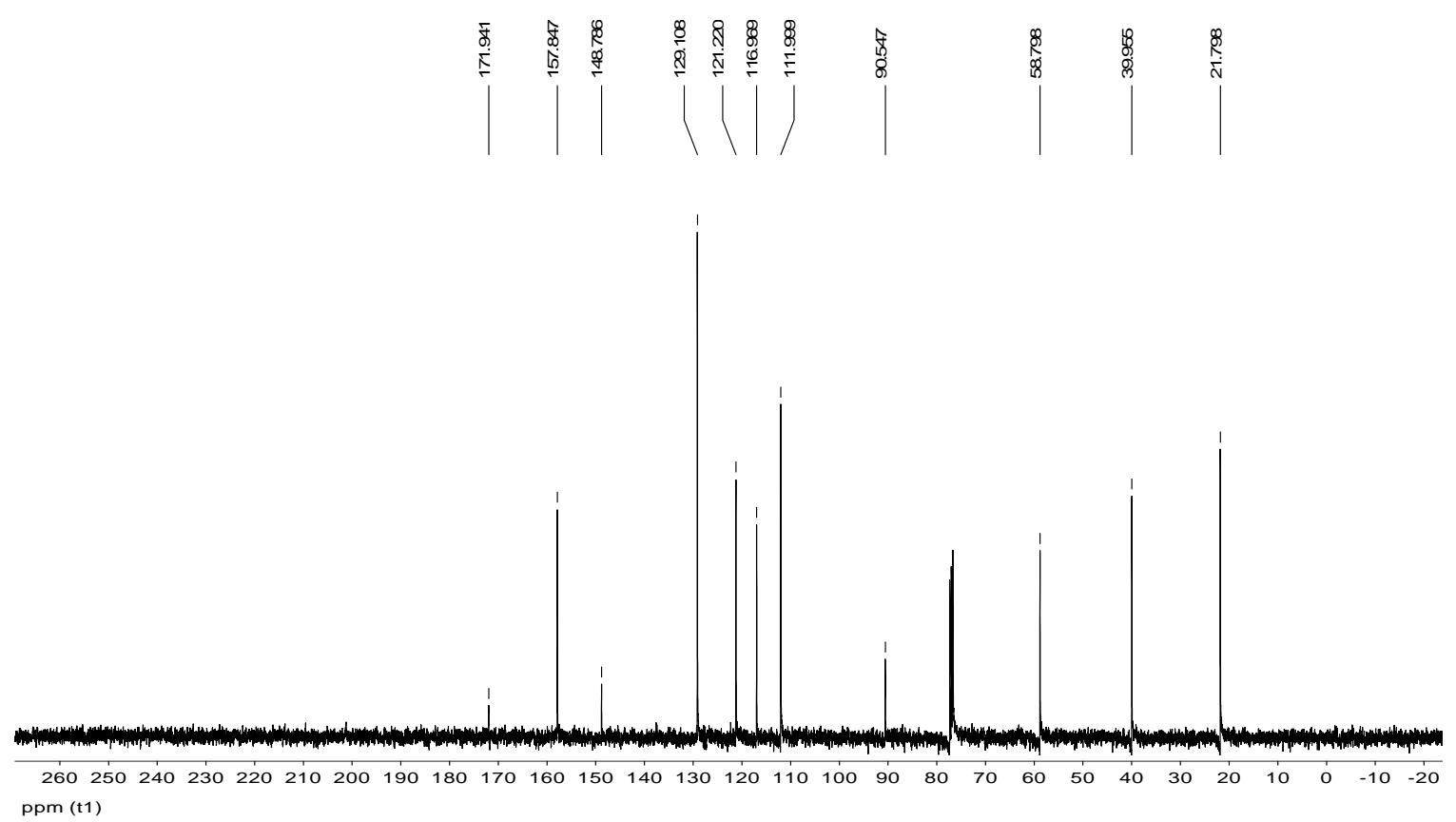




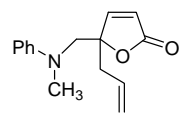

Table 2, entry 12: Purified by chromatography on silica gel $(5: 1 \rightarrow 1: 1$ hexanes/EtOAc); yellow oil: $\mathrm{TLC} \mathrm{R}_{f}=0.35$ (3:1 hexanes/EtOAc); ${ }^{1} \mathrm{H}$ NMR (400 MHz, $\left.\mathrm{CDCl}_{3}\right) \delta 7.31$ (d, $J=5.8 \mathrm{~Hz}, 1 \mathrm{H}), 7.23-7.19(\mathrm{~m}, 2 \mathrm{H}), 6.72(\mathrm{t}, J=7.3 \mathrm{~Hz}, 1 \mathrm{H}), 6.64$ (d, $J=8.3 \mathrm{~Hz}, 2 \mathrm{H}), 5.95(\mathrm{~d}, J=5.6 \mathrm{~Hz}$, 1H), $5.73-5.63(\mathrm{~m}, 1 \mathrm{H}), 5.20-5.15$ (comp, 2H), $3.78(\mathrm{~d}, J=15.7 \mathrm{~Hz}, 1 \mathrm{H}), 3.66(\mathrm{~d}, J=15.7 \mathrm{~Hz}, 1 \mathrm{H})$, 2.96 (s, 3H), $2.66-2.54(\mathrm{~m}, 2 \mathrm{H}) ;{ }^{13} \mathrm{C}$ NMR (100 MHz) $\delta 171.9,156.3,148.8,130.1,129.1,122.3,120.5$, 117.0, 112.0, 92.2, 57.6, 40.1, 39.6; IR (neat) $1756(\mathrm{C}=\mathrm{O}) \mathrm{cm}^{-1}$; HRMS (EI) calcd for $\mathrm{C}_{15} \mathrm{H}_{18} \mathrm{NO}_{2} 244.1338$, found $244.1333(\mathrm{M}+\mathrm{H})$.

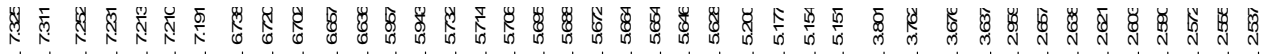

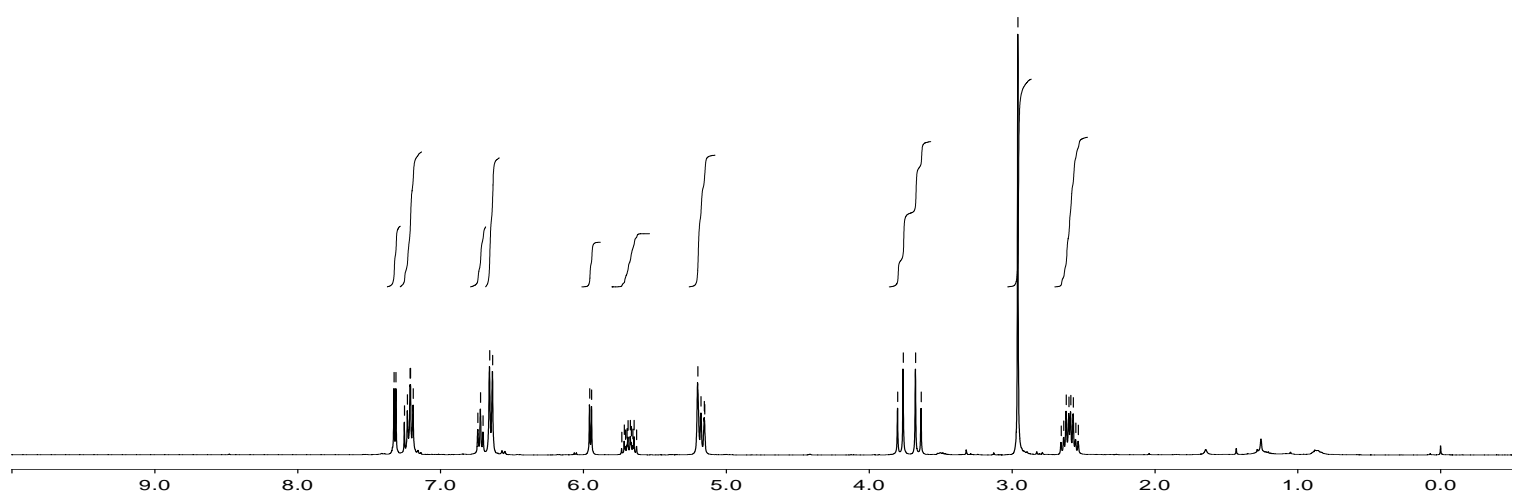

ppm (t1)
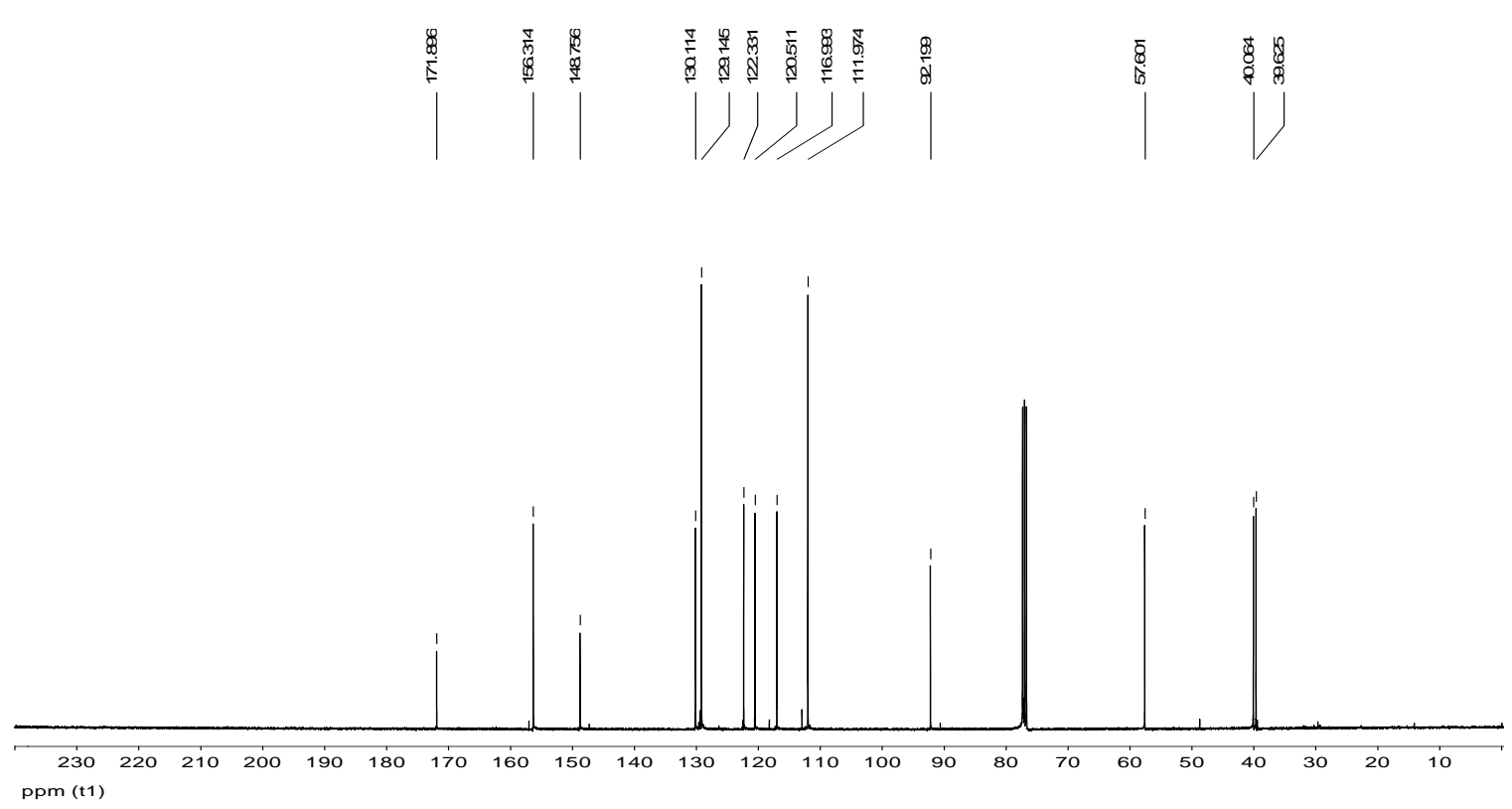